\title{
CONSTRUCTION AND CONVERGENCE ANALYSIS OF CONSERVATIVE SECOND ORDER LOCAL TIME DISCRETISATION FOR LINEAR WAVE EQUATIONS
}

\author{
Juliette Chabassier $^{1}$ And SÉBastien Imperiale ${ }^{2, *}$
}

\begin{abstract}
In this work we present and analyse a time discretisation strategy for linear wave equations that aims at using locally in space the most adapted time discretisation among a family of implicit or explicit centered second order schemes. The proposed family of schemes is adapted to domain decomposition methods such as the mortar element method. They correspond in that case to local implicit schemes and to local time stepping. We show that, if some regularity properties of the solution are satisfied and if the time step verifies a stability condition, then the family of proposed time discretisations provides, in a strong norm, second order space-time convergence. Finally, we provide 1D and 2D numerical illustrations that confirm the obtained theoretical results and we compare our approach on 1D test cases to other existing local time stepping strategies for wave equations.
\end{abstract}

Mathematics Subject Classification. 35L05, 65M12, 65M22, 65M60.

Received July 20, 2020. Accepted June 29, 2021.

\section{INTRODUCTION}

The appropriate time integration of linear systems of ordinary differential equations (ODEs) resulting from the finite element discretisation in space of partial differential equations is of crucial importance to construct efficient numerical solvers. For linear wave equations problems, fully explicit time discretisations perform better than implicit ones in non-stiff situations [9], i.e. when wave propagation occurs in homogeneous media and simple geometries that are quasi-uniformly meshed. However if a strong heterogeneity (high wave speed, low density) is considered, or if the mesh size and quality degenerate locally in space, then explicit methods reach their bottlenecks: the time step of the simulation must be adapted to the local perturbation of the discretisation's parameters, due to the stability condition of the method, namely the CFL condition. In the case of linear wave equations, local time discretisation is a well covered topic that aims at overcoming these bottlenecks. Two main strategies can be distinguished:

Keywords and phrases. Wave equations, time discretisation, converge analysis, local implicit scheme, local time stepping.

1 Team Magique 3D, Inria, E2S UPPA, CNRS, 200 avenue de la vieille tour, 33405 Talence Cedex or avenue de l'université, 64013 Pau Cedex, France.

2 Inria - LMS, École Polytechnique, CNRS - Institut Polytechnique de Paris, 1 rue Honoré d'Estienne d'Orves, 91120 Palaiseau, France.

*Corresponding author: sebastien.imperiale@inria.fr 
- Local implicit time discretisation, see for instance $[7,18,21,30,31,39]$. The strategy is to treat by an implicit time integration scheme the ODEs acting on the degrees of freedom corresponding to the region where the pertubations occur. By doing so, the time step restriction (CFL) is decorrelated from the perturbations. The price to pay is that a (hopefully small) linear problem must be solved at each iteration.

- Local Time Stepping (LTS), see for instance [17,19,22,26,38]. The strategy is to use a first time marching scheme in the whole domain and a second one in the perturbed region. The chosen type of time discretisation used in both regions is often the same but time steps differ: a smaller time step is used locally. One can distinguish non-conservative strategies (see for instance [22]) from conservative strategies. The latter are based upon Leap-Frog schemes and can be separated into two categories depending on how sub-domains are coupled.

- Implicit LTS. A domain decomposition strategy is introduced at the continuous level together with some coupling conditions at the interface of the subdomains (typically by introducing a Lagrange multiplier to enforce in a weak sense those conditions as in the mortar element method, see [42] or [28]). This idea can be traced back to the work of Collino et al. [13,14] and has been pursued and improved in [2,17,33,37,38]. Such strategy is referred as implicit since the treatment of the transmission conditions is done implicitly at the fully discrete level.

- Fully explicit LTS. The decomposition of the domain is done at the discrete level through the use of a discrete restriction operator on the region (and its surrounding) where perturbations occur. The resulting scheme does not introduce transmission conditions in the classical sense but is fully explicit. It has first been proposed in [19] and various extensions exist: Maxwell's equations (see [25]) and multi-level LTS (see [20]). Recently, in [26,27], a proof of space-time convergence is given. It shows that, for the scalar wave equation, a second order space-time convergence holds in the $L^{2}$ norm in space.

In general, the space-time convergence analysis for these numerical methods is not always available, Grote et al. $[26,27]$ being one exception, and although the methods have been implemented in realistic situations as $2 \mathrm{D}$ and $3 \mathrm{D}$ frameworks $[18,21,22]$, their theoretical background does not always rely on a robust analysis. The question of convergence of the method as both the space and time steps vanish together is of crucial importance when dealing with PDEs as linear wave equations, and to this purpose, the energy based analysis has proved very efficient [17] and will be followed in the present work.

In this work we construct and analyse local time discretisations that gather in an original framework both local implicit time discretisation and conservative implicit LTS. Moreover:

- We show that the proposed time discretisations provide, under some regularity and stability conditions, second order space-time convergence, in a strong norm (for scalar wave equations, it provides convergence for the $H^{1}$-norm in space).

- We provide extensive numerical convergence experiments for a 1D scalar wave propagation problem. The results show that our approach provides better space-time convergence properties, in the $H^{1}$-norm, than existing LTS approaches. In particular we study some situations where the LTS of [19] converges in $\Delta t^{3 / 2}$ in $L^{\infty}\left(0, T ; H^{1}(\Omega)\right)$ whereas our approach always provides second order convergence.

The outline of the article is the following. In Section 2 we give all the necessary notations and assumptions related to the discretisation in space of linear conservative wave type problems. Section 3 is devoted to the introduction of a class of time discretisations - parameterised by two polynomial functions $\mathcal{P}_{p}$ and $\mathcal{P}_{k}-$ for which we show stability and second order space-time convergence results under some assumptions on the parameters (i.e. the coefficients of the polynomials $\mathcal{P}_{p}$ and $\mathcal{P}_{k}$ ) and some CFL conditions. In Section 4 we first present two preliminary applications of our discretisation framework. By adequately choosing the polynomial functions $\mathcal{P}_{p}$ and $\mathcal{P}_{k}$ we construct a local implicit time discretisation (Sect. 4.1) and, in Section 4.2, a first local timestepping scheme (with a ratio 2, see Sect. 5.2 for an accurate definition of the term ratio). Finally, in Section 4.3 we propose a strategy to construct general local time-stepping schemes. This strategy is based on the use of Chebychev polynomials (more precisely on Leap-Frog Chebychev method as introduced in [24]). Space-time 
numerical convergence results illustrate the developed theory for 1D test cases in Section 5 and for 2D test cases in Section 6. Finally, in Section 7 we compare our approach algorithmically to other local time stepping strategies: we first compare our approach numerically with the Fully explicit LTS of [19]. Moreover we explain why the proposed schemes can be seen as a generalisation of the ones proposed in [17].

The source code used to obtain convergence curves of Sections 5 and 7 are available as supplementary materials at the web link [44].

\section{SEMI-Discrete WAVE PROPAGATION PROBLEM}

We are interested in the simulation of coupled linear wave propagation problems. The most simple example one could think of is given by the following problem: being given a bounded connected open domain $\Omega$ partitioned as two disjoint connected domains $\Omega_{c}$ and $\Omega_{f}$, find $u_{c}(t) \in H^{1}\left(\Omega_{c}\right)$ and $u_{f}(t) \in H^{1}\left(\Omega_{f}\right)$, for all $t \in[0, T]$ such that

$$
\begin{cases}\partial_{t}^{2} u_{c}-\nabla \cdot \mu_{c} \nabla u_{c}=f_{c} & \text { in } \Omega_{c}, \\ \partial_{t}^{2} u_{f}-\nabla \cdot \mu_{f} \nabla u_{f}=f_{f} & \text { in } \Omega_{f},\end{cases}
$$

with for instance homogeneous Neumann boundary conditions on the domain's boundary

$$
\nabla u_{c} \cdot n=0 \text { on } \partial \Omega_{c} \cap \partial \Omega, \quad \nabla u_{f} \cdot n=0 \text { on } \partial \Omega_{f} \cap \partial \Omega,
$$

and some transmission conditions on the complementary boundary $\Sigma$, that satisfies $\Sigma \cap \partial \Omega=\emptyset$,

$$
u_{c}=u_{f}, \quad \mu_{c} \nabla u_{c} \cdot n=\mu_{f} \nabla u_{f} \cdot n \quad \text { on } \Sigma=\partial \Omega_{c} \cap \partial \Omega_{f},
$$

where $n$ is the outward unitary normal of $\Omega_{c}$. That is completed with initial conditions for $u_{c}$ and $u_{f}$ and their time derivative. The scalar functions $\mu_{c} \in L^{\infty}\left(\Omega_{c}\right)$ and $\mu_{f} \in L^{\infty}\left(\Omega_{f}\right)$ are positive and bounded from below. Such problems find applications in the wave scattering by obstacles and are of interest for modeling non destructive experiments for instance.

\subsection{Continuous abstract formulation and main assumptions}

In the following $q$ stands for either $c$ or $f$.

In this section we formulate the coupled wave propagation in a more abstract framework. To do so we use notations from [16], chapter XVIII, and [6]. We assume given Hilbert spaces $\left(H_{q}, V_{q}\right)$. The space $H_{q}$ is equipped with the scalar product $(\cdot, \cdot)_{q}$, the norm in $H_{q}$ is denoted $|\cdot|_{q}$ whereas the norms on $V_{q}$ is denoted $\|\cdot\|_{q}$. Moreover we assume that $V_{q}$ is dense and continuously embedded in $H_{q}$. We assume given a continuous hermitian bilinear form $a_{q}: V_{q} \times V_{q} \rightarrow \mathbb{R}$ that satisfies for some real positive scalars $c_{q}$ and $C_{q}$,

$$
a_{q}(v, v) \geq 0 \quad \text { and } \quad c_{q}^{2}\|v\|_{q}^{2} \leq C_{q}|v|_{q}+a_{q}(v, v), \quad \forall v \in V_{q} .
$$

We assume also being given another Hilbert space $L$ equipped with the norm $\|\cdot\|_{L}$ as well as a continuous bilinear form $b_{q}(v, \lambda)$ on $V_{q} \times L$. We consider the following abstract wave propagation problem:

Let $f_{c} \in C^{0}\left([0, T], H_{c}\right)$ and $f_{f} \in C^{0}\left([0, T], H_{f}\right)$ be given, find $\left(u_{c}(t), u_{f}(t), \lambda(t)\right) \in V_{c} \times V_{f} \times L$ solution, for all $t \in[0, T]$, to the coupled system of equations

$$
\begin{cases}\frac{\mathrm{d}^{2}}{\mathrm{~d} t^{2}}\left(u_{c}, v_{c}\right)_{c}+a_{c}\left(u_{c}, v_{c}\right)+b_{c}\left(v_{c}, \lambda\right)=\left(f_{c}, v_{c}\right)_{c} & \forall v_{c} \in V_{c}, \\ \frac{\mathrm{d}^{2}}{\mathrm{~d} t^{2}}\left(u_{f}, v_{f}\right)_{f}+a_{f}\left(u_{f}, v_{f}\right)-b_{f}\left(v_{f}, \lambda\right)=\left(f_{f}, v_{f}\right)_{f} & \forall v_{f} \in V_{f}, \\ b_{c}\left(u_{c}, \mu\right)=b_{f}\left(u_{f}, \mu\right) & \forall \mu \in L,\end{cases}
$$


that is completed with initial conditions for $u_{c}$ and $u_{f}$ and their time derivative. Note that the scalar wave equation problem (2.1)-(2.5) enters the abstract framework presented above by choosing

$$
H_{q}=L^{2}\left(\Omega_{q}\right), \quad V_{q}=\left\{v \in H^{1}\left(\Omega_{q}\right)\right\}, \quad L=H^{-1 / 2}(\Sigma),
$$

where $H_{q}$ is equipped with the standard $L^{2}$ scalar product, and for all $u$ and $v$ in $V_{q}$ and for all $\lambda$ in $H^{-1 / 2}(\Sigma)$

$$
a_{q}(u, v)=\left(\mu_{q} \nabla u, \nabla v\right)_{q}, \quad b_{q}(v, \lambda)=\left\langle\left. v\right|_{\Sigma}, \lambda\right\rangle_{H^{1 / 2}(\Sigma), H^{-1 / 2}(\Sigma)} .
$$

However, the setting we consider is rather general and for instance elastodynamics equations could also enter the abstract framework by writing transmission problems (continuity of displacements and stresses) and using vectorial forms of all the spaces and scalar products introduced. Moreover the abstract structure is also adapted to the domain decomposition method with overlapping introduced in [1] to deal with scattering problems in transient acoustics.

System (2.3) can be rewritten in a more compact form using the following notation: bold letters are used to define unknowns in $\boldsymbol{V}=V_{c} \times V_{f}$, e.g., $\boldsymbol{u}=\left(u_{c}, u_{f}\right)$ and we introduce the bilinear forms

$$
a(\boldsymbol{u}, \boldsymbol{v}):=a_{c}\left(u_{c}, v_{c}\right)+a_{f}\left(u_{f}, v_{f}\right), \quad(\boldsymbol{u}, \boldsymbol{v}):=\left(u_{c}, v_{c}\right)_{c}+\left(u_{f}, v_{f}\right)_{f}
$$

as well as $b(\boldsymbol{v}, \lambda):=b_{c}\left(v_{c}, \lambda\right)-b_{f}\left(v_{f}, \lambda\right)$. Then (2.3) can be recast as: find $(\boldsymbol{u}(t), \lambda(t)) \in \boldsymbol{V} \times L$ solution to

$$
\begin{cases}\frac{\mathrm{d}^{2}}{\mathrm{~d} t^{2}}(\boldsymbol{u}, \boldsymbol{v})+a(\boldsymbol{u}, \boldsymbol{v})+b(\boldsymbol{v}, \lambda)=(\boldsymbol{f}, \boldsymbol{v}) & \boldsymbol{v} \in \boldsymbol{V}, \\ b(\boldsymbol{u}, \mu)=0 & \mu \in L .\end{cases}
$$

We complete (2.4) with initial conditions

$$
\boldsymbol{u}(0)=\boldsymbol{u}_{0}, \quad \frac{\mathrm{d}}{\mathrm{d} t} \boldsymbol{u}(0)=\boldsymbol{u}_{1} \quad \text { in } \boldsymbol{V}, \quad b\left(\boldsymbol{u}_{0}, \mu\right)=b\left(\boldsymbol{u}_{1}, \mu\right)=0, \quad \mu \in L .
$$

Existence and uniqueness results for this abstract problem rely on the assumption that a so-called inf-sup condition holds. More precisely we assume that there exists $c_{b}>0$ such that

$$
\inf _{\lambda \in L} \sup _{\boldsymbol{v} \in \boldsymbol{V}} \frac{b(\boldsymbol{v}, \lambda)}{\|\lambda\|_{L}\|\boldsymbol{v}\|} \geq c_{b}
$$

where $\|\boldsymbol{v}\|^{2}=\left\|v_{c}\right\|_{c}^{2}+\left\|v_{f}\right\|_{f}^{2}$ (similarly we denote by $|\cdot|$ the composite norm in $\boldsymbol{H}=H_{c} \times H_{f}$ ). Notice that this condition is the same encountered in steady domain decomposition problems. We refer the reader to Chap. 7 of [5] for a proof. We do not provide here proofs of existence and uniqueness for such problems - they rely on energy analysis and/or Laplace transform - and refer, for instance to the work of $[1,3]$ for related analysis. See also the Appendix A of this manuscript for more details on the matter. We assume that the solution has the following properties

Assumption 2.1. There exists a unique

$$
(\boldsymbol{u}, \lambda) \in C^{4}([0, T] ; \boldsymbol{H}) \cap C^{3}([0, T] ; \boldsymbol{V}) \times C^{2}([0, T] ; L)
$$

solution to problem (2.4) and (2.5).

\subsection{Discretisation in space, main assumptions and stability estimates}

We introduce the family of finite dimensional Hilbert spaces $\left\{V_{q, h}\right\}_{h>0}$ with $V_{q, h} \subset V_{q}$ and $L_{h} \subset L$. The space $V_{q, h}$ (resp. $\left.L_{h}\right)$ is equipped with the scalar product $(,)_{q}\left(\operatorname{resp}(,)_{L}\right)$. As usual, the subscript $h$ is devoted 
to tend to 0 and represents an approximation parameter of $V_{q, h}$ to $V_{q}$ and $L_{h}$ to $L$. For each $h$ we define the operator $A_{q, h}$ as $A_{q, h}: V_{q, h} \rightarrow V_{q, h}$ and

$$
A_{q, h}: u_{h} \mapsto A_{q, h} u_{h} \quad \text { such that } \quad\left(A_{q, h} u_{h}, v_{h}\right)_{q}=a_{q}\left(u_{h}, v_{h}\right), \quad \forall v_{h} \in V_{q, h} .
$$

Inequality (2.2) implies that the operator $A_{q, h}$ is self-adjoint and positive semi-definite. Its spectrum, denoted $\operatorname{Sp}\left(A_{q, h}\right)$, is composed by a finite number of non-negative eigenvalues. The spectral radius is defined as the maximum eigenvalue in the set $\operatorname{Sp}\left(A_{q, h}\right)$, i.e.,

$$
\rho_{q, h}:=\max \operatorname{Sp}\left(A_{q, h}\right) .
$$

We also introduce the operators $B_{q, h}: V_{q, h} \mapsto L_{h}$ and $B_{q, h}^{t}: L_{h} \mapsto V_{q, h}$ as

$$
\left(B_{q, h} v_{q, h}, \lambda_{h}\right)_{L}=\left(B_{q, h}^{t} \lambda_{h}, v_{q, h}\right)_{q}:=b_{q}\left(v_{q, h}, \lambda_{h}\right), \quad \forall v_{q, h} \in V_{q, h} \text { and } \forall \lambda_{h} \in L_{h} .
$$

As done previously we define $\boldsymbol{V}_{h}=V_{c, h} \times V_{f, h}$ and represent by bold letters unknowns in $\boldsymbol{V}_{h}$. The semi-discrete equation we consider reads:

Let $\boldsymbol{f}_{h} \in C^{0}\left([0, T], V_{q, h}\right)$ be given: find $\left(\boldsymbol{u}_{h}(t), \lambda_{h}(t)\right) \in \boldsymbol{V}_{h} \times L_{h}$ and solution, for all $t \in[0, T]$, of

$$
\left\{\begin{array}{lll}
\frac{\mathrm{d}^{2}}{\mathrm{~d} t^{2}} u_{c, h}+A_{c, h} u_{c, h}+B_{c, h}^{t} \lambda_{h}=f_{c, h} & \text { in } V_{c, h}, & (a) \\
\frac{\mathrm{d}^{2}}{\mathrm{~d} t^{2}} u_{f, h}+A_{f, h} u_{f, h}-B_{f, h}^{t} \lambda_{h}=f_{f, h} & \text { in } V_{f, h}, & (b) \\
B_{c, h} u_{c, h}=B_{f, h} u_{f, h} & \text { in } L_{h}, & (c)
\end{array}\right.
$$

together with the initial conditions

$$
\boldsymbol{u}_{h}(0)=\boldsymbol{u}_{0, h}, \quad \frac{\mathrm{d}}{\mathrm{d} t} \boldsymbol{u}_{h}(0)=\boldsymbol{u}_{1, h} \quad \text { in } \boldsymbol{V}_{h}, \quad b\left(\boldsymbol{u}_{0, h}, \mu\right)=b\left(\boldsymbol{u}_{1, h}, \mu\right)=0, \quad \mu \in L_{h} .
$$

In the rest of the work we assume that the following discrete inf-sup condition holds

$$
\inf _{\lambda_{h} \in L_{h}} \sup _{\boldsymbol{v}_{h} \in \boldsymbol{V}_{h}} \frac{b\left(\boldsymbol{v}_{h}, \lambda_{h}\right)}{\left\|\lambda_{h}\right\|_{L}\left\|\boldsymbol{v}_{h}\right\|} \geq C_{b}
$$

where $C_{b}$ is independent of $h$. Since discretisation by finite elements of wave equations is now a well understood subject, most of the difficulty in constructing System (2.8) is to choose $L_{h}$ such that (2.10) holds. In fact, (2.10) is not a consequence of (2.6) and this question requires dedicated analysis. On this specific topic, we refer the reader to $[4,28,41,42]$ for reference work concerning the mortar finite element method, that is a domain decomposition method without overlapping, and to [1], for a work concerning a domain decomposition method with overlapping.

Assumption 2.2. There exists a unique solution $\left(\boldsymbol{u}_{h}, \lambda_{h}\right)$ to (2.8), it satisfies $\boldsymbol{u}_{h} \in C^{4}\left([0, T] ; \boldsymbol{V}_{h}\right)$ and $\lambda_{h} \in$ $C^{2}\left([0, T] ; L_{h}\right)$ as well as the estimate

$$
\sum_{m=1}^{4} \sup _{t \in[0, T]}\left|\frac{\mathrm{d}^{m}}{\mathrm{~d} t^{m}} \boldsymbol{u}_{h}(t)\right|+\sum_{m=0}^{3} \sup _{t \in[0, T]}\left\|\frac{\mathrm{d}^{m}}{\mathrm{~d} t^{m}} \boldsymbol{u}_{h}(t)\right\| \leq C
$$

where $C>0$ is independent of $h$. 
To state a semi-discrete convergence result we introduce the discrepancy error

$$
\boldsymbol{e}_{h}(t)=\boldsymbol{u}(t)-\boldsymbol{u}_{h}(t)
$$

Since the main focus of this work is the analysis of time discretisations of the semi-discrete problem (2.8), we postponed the proof of the theorem below to Appendix A.

Theorem 2.3 (Convergence of the semi-discrete problem). Let Assumptions 2.1 and 2.2 hold. Let us define the error term,

$$
\begin{aligned}
\delta_{h}= & \left\|\boldsymbol{u}_{h, 0}-\boldsymbol{u}_{0}\right\|+\left|\boldsymbol{u}_{h, 1}-\boldsymbol{u}_{1}\right| \\
& +\sup _{t \in[0, T]}\left(\left|\boldsymbol{f}_{h}(t)-\boldsymbol{f}(t)\right|+\sum_{m=0}^{2} \inf _{\boldsymbol{v}_{h} \in \boldsymbol{V}_{h}}\left\|\boldsymbol{v}_{h}-\frac{\mathrm{d}^{m}}{\mathrm{~d} t^{m}} \boldsymbol{u}(t)\right\|+\sum_{m=0}^{2} \inf _{\mu_{h} \in L_{h}}\left\|\mu_{h}-\frac{\mathrm{d}^{m}}{\mathrm{~d} t^{m}} \lambda(t)\right\|_{L}\right),
\end{aligned}
$$

then, there exists a scalar $C$ independent of $h$ such that

$$
\sup _{t \in[0, T]}\left\|\boldsymbol{e}_{h}(t)\right\| \leq C \delta_{h}
$$

Remark 2.4. For simplicity of analysis we have assumed that bilinear forms are evaluated exactly. However the results presented in this work could be extended to take into account the use of quadrature formulae for the computation of space integrals. Moreover, numerical convergence results will be presented using the mass-lumping strategy which is obtained using specific quadrature formulae (see [12] or [10]).

\section{Time Discretisation}

The schemes we construct here can be seen as perturbations, for small time step $\Delta t$, of the standard centered two-steps discretisation of system (2.8). The perturbations are defined by two polynomials $\mathcal{P}_{k}(x)$ and $\mathcal{P}_{p}(x)$ to be determined. In this section we first construct time discretisation with the minimum assumptions concerning the properties that should be satisfied by the polynomials and then state a space-time convergence result. In Section 4 some examples are given that show how efficient local time discretisation can be constructed from adequate definition of $\mathcal{P}_{k}(x)$ and $\mathcal{P}_{p}(x)$.

\subsection{Introduction of local time discretisations}

We define the sequences $\left\{\boldsymbol{u}_{h}^{n}=\left(u_{c, h}^{n}, u_{f, h}^{n}\right)\right\}$ and $\left\{\lambda_{h}^{n}\right\}$ as the approximations of $\boldsymbol{u}_{h}(t)$ and $\lambda_{h}(t)$ at time $t=n \Delta t$ for a time step $\Delta t>0$, and $n \in\{1,2, \ldots, N\}$. We define the final time of computation as $T=N \Delta t$. These sequences are constructed by solving the following problem:

Let $\boldsymbol{f}_{h} \in C^{0}\left([0, T], \boldsymbol{V}_{h}\right)$ be given, find $\left(\left\{\boldsymbol{u}_{h}^{n}\right\},\left\{\lambda_{h}^{n}\right\}\right)$ solution to

$$
\begin{cases}\frac{u_{c, h}^{n+1}-2 u_{c, h}^{n}+u_{c, h}^{n-1}}{\Delta t^{2}}+A_{c, h} u_{c, h}^{n}+B_{c, h}^{t} \lambda_{h}^{n}=f_{c, h}\left(t^{n}\right) & \text { in } V_{c, h}, \quad(a) \\ \mathcal{P}_{k}\left(\Delta t^{2} A_{f, h}\right) \frac{u_{f, h}^{n+1}-2 u_{f, h}^{n}+u_{f, h}^{n-1}}{\Delta t^{2}} & \\ \quad+\mathcal{P}_{p}\left(\Delta t^{2} A_{f, h}\right)\left(A_{f, h}\left\{u_{f, h}^{n}\right\}_{1 / 4}-B_{f, h}^{t} \lambda_{h}^{n}-f_{f, h}\left(t^{n}\right)\right)=0 & \text { in } V_{f, h},(b) \\ B_{c, h} u_{c, h}^{n}=B_{f, h} u_{f, h}^{n} & \text { in } L_{h}, \quad(c)\end{cases}
$$

where

$$
\left\{u_{f, h}^{n}\right\}_{1 / 4}=\frac{u_{f, h}^{n+1}+2 u_{f, h}^{n}+u_{f, h}^{n-1}}{4},
$$


with the initial conditions

$$
\boldsymbol{u}_{h}^{0}=\boldsymbol{u}_{0, h}, \quad \boldsymbol{u}_{h}^{1}=\boldsymbol{u}_{0, h}+\Delta t \boldsymbol{u}_{1, h}+\frac{\Delta t^{2}}{2} \frac{\mathrm{d}^{2}}{\mathrm{~d} t^{2}} \boldsymbol{u}_{h}(0) \quad \text { in } \boldsymbol{V}_{h} .
$$

We give more detail in Remark 3.5 on how the term $\boldsymbol{u}_{h}^{1}$ can be computed.

The scheme (2.3) is consistent only if some conditions are satisfied on the polynomials $\mathcal{P}_{k}(x)$ and $\mathcal{P}_{p}(x)$. Since we want to construct perturbations, for small $\Delta t$, of the standard centered scheme it seems natural to do the the following hypothesis.

\section{Assumption 3.1.}

$$
\mathcal{P}_{k}(0)=\mathcal{P}_{p}(0)=1
$$

For stability reasons the time step $\Delta t$ can not be chosen arbitrarily. A so called CFL-condition has to be satisfied to obtain a stable scheme. In our case it corresponds to the assumption that follows.

Assumption 3.2. The following CFL-condition holds: there exists $0<\alpha \leq 1$ such that

$$
\Delta t=\alpha \frac{2}{\sqrt{\rho_{c, h}}}
$$

and

$$
\mathcal{P}_{k}(x) \geq 0, \quad \mathcal{P}_{p}(x)>0, \quad \forall x \in\left[0, \Delta t^{2} \rho_{f, h}\right] .
$$

Note that the spectral radius $\rho_{c, h}$ of equation $(3.3)$ is $\mathcal{O}\left(h^{-2}\right)$ for regular discretization when standard finite element methods are used, leading to a classical hyperbolic-type CFL condition. Note also that since

$$
\Delta t^{2} \rho_{f, h}=4 \alpha^{2} \frac{\rho_{f, h}}{\rho_{c, h}},
$$

and because of Assumption 3.1, we know that there exists $\Delta t$ small enough or equivalently $\alpha$ small enough, such that (3.4) is satisfied for any fixed $h$. As shown later, these conditions ensure the positivity of a preserved discrete energy.

We describe now more in detail an algorithm that computes the solution to (3.1). At each iteration, one needs to compute the Lagrange multiplier $\lambda_{h}^{n}$ first, then compute $u_{f, h}^{n+1}$ and $u_{c, h}^{n+1}$. More precisely, using the property that

$$
\left\{u_{f, h}^{n}\right\}_{1 / 4}=u_{f, h}^{n}+\frac{\Delta t^{2}}{4} \frac{u_{f, h}^{n+1}-2 u_{f, h}^{n}+u_{f, h}^{n-1}}{\Delta t^{2}}
$$

we can re-write equation (3.1b) in the following form

$$
\frac{u_{f, h}^{n+1}-2 u_{f, h}^{n}+u_{f, h}^{n-1}}{\Delta t^{2}}+D_{f, h}^{-1} \mathcal{P}_{p}\left(\Delta t^{2} A_{f, h}\right)\left(A_{f, h} u_{f, h}^{n}-B_{f, h}^{t} \lambda_{h}^{n}-f_{f, h}\left(t^{n}\right)\right)=0,
$$

with

$$
D_{f, h}:=\mathcal{P}_{k}\left(\Delta t^{2} A_{f, h}\right)+\frac{\Delta t^{2}}{4} \mathcal{P}_{p}\left(\Delta t^{2} A_{f, h}\right) A_{f, h} .
$$

Note that $D_{f, h}$ is a positive symmetric operator - hence invertible - if equation (3.4) holds. We now use a Schur complement technique: applying the operator $B_{f, h}$ to equation (3.7), applying the operator $B_{c, h}$ to (3.1a), we obtain by subtraction and thanks to (3.1c) the following system for the Lagrange multiplier $\lambda_{h}^{n}$

$$
\begin{aligned}
\left(B_{c, h} B_{c, h}^{t}+B_{f, h} D_{f, h}^{-1} \mathcal{P}_{p}\left(\Delta t^{2} A_{f, h}\right) B_{f, h}^{t}\right) \lambda_{h}^{n}= & B_{c, h} f_{c, h}\left(t^{n}\right)-B_{c, h} A_{c, h} u_{c, h}^{n} \\
& +B_{f, h} D_{f, h}^{-1} \mathcal{P}_{p}\left(\Delta t^{2} A_{f, h}\right)\left(A_{f, h} u_{f, h}^{n}-f_{f, h}\left(t^{n}\right)\right) .
\end{aligned}
$$

The well-posedness of the above problem is a consequence of the surjectivity of either $B_{c, h}$ or $B_{f, h}$ which is a consequence of the inf-sup condition (2.10). Assuming that $u_{f, h}^{n}$ and $u_{c, h}^{n}$ are known, then $\lambda_{h}^{n}$ can be computed using (3.8), it follows that $u_{f, h}^{n+1}$ and $u_{c, h}^{n+1}$ can be computed using respectively (3.7) and (3.1a). 
Remark 3.3. A drastic simplification occurs when

$$
\mathcal{P}_{k}(x)=1-x \frac{\mathcal{P}_{p}(x)}{4} .
$$

In that case $D_{f, h}$ is the identity operator on $V_{f, h}$ and the volumic unknown $u_{f, h}^{n+1}$ can be explicitly updated.

Remark 3.4. With the choice $\mathcal{P}_{p}(x)=1$ and $\mathcal{P}_{k}(x)=1-x / 4$ we obtain a standard coupled explicit leap-frog schemes. It is not difficult to prove that the corresponding stability condition reads

$$
\Delta t^{2} \leq \min \left(\frac{4}{\rho_{c, h}}, \frac{4}{\rho_{f, h}}\right)
$$

Condition (3.10) is penalizing since it depends in the same way in $\rho_{c, h}$ and $\rho_{f, h}$ but the latter can be large compared to $\rho_{c, h}$.

Remark 3.5. The computation of the initial data using formula (3.2) involves the computation of the term $d_{t}^{2} \boldsymbol{u}_{h}(0)$. This term is obtained by evaluating (2.8) at time $t=0$. More precisely, we have

$$
\left(\begin{array}{ccc}
I_{c, h} & 0 & B_{c, h}^{t} \\
0 & I_{f, h} & -B_{f, h}^{t} \\
-B_{c, h} & B_{f, h} & 0
\end{array}\right)\left(\begin{array}{c}
d_{t}^{2} u_{c, h}(0) \\
d_{t}^{2} u_{f, h}(0) \\
\lambda_{h}(0)
\end{array}\right)=\left(\begin{array}{c}
f_{c, h}(0)-A_{c, h} u_{c, 0, h} \\
f_{f, h}(0)-A_{f, h} u_{f, 0, h} \\
0
\end{array}\right)
$$

Using a Schur complement, the Lagrange multiplier is given

$$
\left(B_{c, h} B_{c, h}^{t}+B_{f, h} B_{f, h}^{t}\right) \lambda_{h}(0)=B_{c, h}\left(f_{c, h}(0)-A_{c, h} u_{c, 0, h}\right)-B_{f, h}\left(f_{f, h}-A_{f, h} u_{f, 0, h}\right) .
$$

As already mentioned the well-posedness of the above problem is a consequence of the surjectivity of either $B_{c, h}$ or $B_{f, h}$ which is a consequence of the inf-sup condition (2.10). Once $\lambda_{h}(0)$ is computed, the value of $d_{t}^{2} \boldsymbol{u}_{h}(0)$ is obtained easily from (3.11). Finally note that the initial data satisfy by construction the constraints $B_{c, h} u_{c, h}^{0}=B_{f, h} u_{f, h}^{0}$ and $B_{c, h} u_{c, h}^{1}=B_{f, h} u_{f, h}^{1}$.

\subsection{Space-time convergence analysis}

We define the error terms $\boldsymbol{e}_{h}^{n}=\left(e_{c, h}^{n}, e_{f, h}^{n}\right)$ and $\ell_{h}^{n}$ as

$$
\boldsymbol{e}_{h}^{n}=\boldsymbol{u}_{h}\left(t^{n}\right)-\boldsymbol{u}_{h}^{n} \quad \text { and } \quad \ell_{h}^{n}=\lambda_{h}\left(t^{n}\right)-\lambda_{h}^{n} .
$$

In this section we show that the terms $\boldsymbol{e}_{h}^{n}$ tends to 0 as $h$ and $\Delta t$ go to 0 . More precisely we show that under Assumptions 3.1, 3.2 and 3.7 (given below) we obtain a uniform estimation with respect to $\Delta t$ and $h$ in the norm $L^{\infty}(0, T ; \boldsymbol{V})$ of the error in $O\left(\Delta t^{2}\right)+O\left(\delta_{h}\right)$. The section is organized as follows

- Definition of the consistency errors: we write a system of equations for the sequence $e_{c, h}^{n}, e_{f, h}^{n}$ and $\ell_{h}^{n}$ that is similar to (3.1) with source terms that correspond to consistency errors that we will then specify.

- Energy identity for the error equation: we proceed by energy analysis and write an energy identity satisfied by the error terms $e_{c, h}^{n}$ and $e_{f, h}^{n}$. The introduced energy is positive under the CFL-condition of Assumption 3.2.

- Stability result: we prove that the energy associated to the error $\left\{\boldsymbol{e}_{h}^{n}\right\}$ is stable. To do so we use a discrete by-parts integration and a discrete energy analysis including the use of a discrete Gronwall's lemma.

- Space-time convergence results: using Theorem 2.3, we deduce space-time convergence results in the norm $L^{\infty}(0, T ; \boldsymbol{V})$, 


\subsubsection{Definition of the consistency errors.}

We always assume that Assumption 2.2 holds and therefore that the solution is sufficiently smooth, in particular,

$$
\boldsymbol{u}_{h} \in C^{4}\left([0, T] ; \boldsymbol{V}_{h}\right)
$$

With this assumption, all the manipulations and expression used below make sense in a standard continuous setting. Using equations (2.8) and (3.1) we obtain, for $n \in\{1,2, \ldots, N-1\}$,

$$
\begin{cases}\frac{e_{c, h}^{n+1}-2 e_{c, h}^{n}+e_{c, h}^{n-1}}{\Delta t^{2}}+A_{c, h} e_{c, h}^{n}+B_{c, h}^{t} \ell_{h}^{n}=r_{c, h}^{n} & \text { in } V_{c, h}, \\ \mathcal{P}_{k}\left(\Delta t^{2} A_{f, h}\right) \frac{e_{f, h}^{n+1}-2 e_{f, h}^{n}+e_{f, h}^{n-1}}{\Delta t^{2}}+\mathcal{P}_{p}\left(\Delta t^{2} A_{f, h}\right)\left(A_{f, h}\left\{e_{f, h}^{n}\right\}_{1 / 4}-B_{f, h}^{t} \ell_{h}^{n}\right)=r_{f, h}^{n} & \text { in } V_{f, h}, \\ B_{c, h} e_{c, h}^{n}=B_{f, h} e_{f, h}^{n} & \text { in } L_{h}\end{cases}
$$

with the consistency errors given by

$$
r_{c, h}^{n}=\frac{u_{c, h}\left(t^{n+1}\right)-2 u_{c, h}\left(t^{n}\right)+u_{c, h}\left(t^{n-1}\right)}{\Delta t^{2}}+A_{c, h} u_{c, h}\left(t^{n}\right)+B_{c, h}^{t} \lambda_{h}\left(t^{n}\right)-f_{c, h}\left(t^{n}\right)
$$

and

$$
\begin{aligned}
r_{f, h}^{n}= & \mathcal{P}_{k}\left(\Delta t^{2} A_{f, h}\right) \frac{u_{f, h}\left(t^{n+1}\right)-2 u_{f, h}\left(t^{n}\right)+u_{f, h}\left(t^{n-1}\right)}{\Delta t^{2}} \\
& +\mathcal{P}_{p}\left(\Delta t^{2} A_{f, h}\right)\left(A_{f, h}\left\{u_{f, h}\left(t^{n}\right)\right\}_{1 / 4}-B_{f, h}^{t} \lambda_{h}\left(t^{n}\right)-f_{f, h}\left(t^{n}\right)\right) .
\end{aligned}
$$

Standard Taylor expansions allow us to simplify equations (3.13) and (3.14). There exist intermediate times $\left(t^{n, \odot}, t^{n, \boldsymbol{\bullet}}, t^{n, \boldsymbol{\bullet}}\right)$ with

$$
t^{n-1} \leq t^{n, \odot}, t^{n, \infty}, t^{n, \boldsymbol{\infty}} \leq t^{n+1}
$$

such that, using equation (2.8a),

$$
r_{c, h}^{n}=\frac{\Delta t^{2}}{12} \frac{\mathrm{d}^{4}}{\mathrm{~d} t^{4}} u_{c, h}\left(t^{n, \odot}\right)
$$

and

$$
\begin{aligned}
r_{f, h}^{n}= & \mathcal{P}_{k}\left(\Delta t^{2} A_{f, h}\right) \frac{\mathrm{d}^{2}}{\mathrm{~d} t^{2}} u_{f, h}\left(t^{n}\right)+\mathcal{P}_{p}\left(\Delta t^{2} A_{f, h}\right)\left(A_{f, h} u_{f, h}\left(t^{n}\right)-B_{f, h}^{t} \lambda_{h}\left(t^{n}\right)-f_{f, h}\left(t^{n}\right)\right) \\
& +\frac{\Delta t^{2}}{12} \mathcal{P}_{k}\left(\Delta t^{2} A_{f, h}\right) \frac{\mathrm{d}^{4}}{\mathrm{~d} t^{4}} u_{f, h}\left(t^{n, \boldsymbol{\uparrow}}\right)+\frac{\Delta t^{2}}{4} \mathcal{P}_{p}\left(\Delta t^{2} A_{f, h}\right) A_{f, h} \frac{\mathrm{d}^{2}}{\mathrm{~d} t^{2}} u_{f, h}\left(t^{n, \boldsymbol{中}}\right) .
\end{aligned}
$$

Then using equation (2.8b) one can further simplify $r_{f, h}^{n}$ as

$$
\begin{aligned}
r_{f, h}^{n}= & \left(\mathcal{P}_{k}\left(\Delta t^{2} A_{f, h}\right)-\mathcal{P}_{p}\left(\Delta t^{2} A_{f, h}\right)\right) \frac{\mathrm{d}^{2}}{\mathrm{~d} t^{2}} u_{f, h}\left(t^{n}\right) \\
& +\frac{\Delta t^{2}}{12} \mathcal{P}_{k}\left(\Delta t^{2} A_{f, h}\right) \frac{\mathrm{d}^{4}}{\mathrm{~d} t^{4}} u_{f, h}\left(t^{n, \boldsymbol{\uparrow}}\right)+\frac{\Delta t^{2}}{4} \mathcal{P}_{p}\left(\Delta t^{2} A_{f, h}\right) A_{f, h} \frac{\mathrm{d}^{2}}{\mathrm{~d} t^{2}} u_{f, h}\left(t^{n, \boldsymbol{\bullet}}\right) .
\end{aligned}
$$

If Assumptions 3.1 and 3.2 hold then there exists a rational function $\mathcal{Q}$ such that

$$
\left(\mathcal{P}_{k}\left(\Delta t^{2} A_{f, h}\right)-\mathcal{P}_{p}\left(\Delta t^{2} A_{f, h}\right)\right) \frac{\mathrm{d}^{2}}{\mathrm{~d} t^{2}} u_{f, h}\left(t^{n}\right)=\Delta t^{2} \mathcal{P}_{p}\left(\Delta t^{2} A_{f, h}\right) \mathcal{Q}\left(\Delta t^{2} A_{f, h}\right) A_{f, h} \frac{\mathrm{d}^{2}}{\mathrm{~d} t^{2}} u_{f, h}\left(t^{n}\right)
$$


where $\mathcal{Q}(x)$ is given by

$$
\mathcal{Q}(x):=\mathcal{P}_{p}^{-1}(x) \frac{\mathcal{P}_{k}(x)-\mathcal{P}_{p}(x)}{x} .
$$

The consistency error $r_{f, h}^{n}$ has then the final expression

$$
\begin{aligned}
r_{f, h}^{n}= & \frac{\Delta t^{2}}{12} \mathcal{P}_{k}\left(\Delta t^{2} A_{f, h}\right) \frac{\mathrm{d}^{4}}{\mathrm{~d} t^{4}} u_{f, h}\left(t^{n, \boldsymbol{\uparrow}}\right)+\frac{\Delta t^{2}}{4} \mathcal{P}_{p}\left(\Delta t^{2} A_{f, h}\right) A_{f, h} \frac{\mathrm{d}^{2}}{\mathrm{~d} t^{2}} u_{f, h}\left(t^{n, \boldsymbol{\kappa}}\right) \\
& +\Delta t^{2} \mathcal{P}_{p}\left(\Delta t^{2} A_{f, h}\right) \mathcal{Q}\left(\Delta t^{2} A_{f, h}\right) A_{f, h} \frac{\mathrm{d}^{2}}{\mathrm{~d} t^{2}} u_{f, h}\left(t^{n}\right) .
\end{aligned}
$$

\subsubsection{Energy identity for the error equation}

To obtain an energy identity on the error equations (3.12)-(3.15)-(3.17) we use a standard discrete energy technique. The main ingredients of the strategy is to observe that, if Assumption 3.2 holds then

$$
I_{c, h}-\frac{\Delta t^{2}}{4} A_{c, h}
$$

is a non-negative symmetric operator. Moreover, with the same assumption, if we introduce the following notation,

$$
\mathcal{R}(x):=\mathcal{P}_{p}(x)^{-1} \mathcal{P}_{k}(x)
$$

then $\mathcal{R}\left(\Delta t^{2} A_{f, h}\right)$ is well defined and is a non-negative symmetric operator. Note that from (3.18) and (3.16) we deduce that

$$
\mathcal{Q}(x):=\frac{\mathcal{R}(x)-1}{x} .
$$

After standard algebraic manipulations (similar to the computations done in [7]) one can show the following lemma.

Lemma 3.6. Let Assumption 3.1 and 3.2 hold. Then, for $n \in\{1,2, \ldots, N-1\}$,

$$
\frac{\mathcal{E}_{c, h}^{n+1 / 2}-\mathcal{E}_{c, h}^{n-1 / 2}}{\Delta t}+\frac{\mathcal{E}_{f, h}^{n+1 / 2}-\mathcal{E}_{f, h}^{n-1 / 2}}{\Delta t}=\left(r_{c, h}^{n}, \frac{e_{c, h}^{n+1}-e_{c, h}^{n-1}}{2 \Delta t}\right)_{c}+\left(r_{f, h}^{n}, \mathcal{P}_{p}\left(\Delta t^{2} A_{f, h}\right)^{-1} \frac{e_{f, h}^{n+1}-e_{f, h}^{n-1}}{2 \Delta t}\right)_{f},
$$

with

$$
\mathcal{E}_{c, h}^{n+1 / 2}=\frac{1}{2}\left|\left(I_{c, h}-\frac{\Delta t^{2}}{4} A_{c, h}\right)^{\frac{1}{2}} \frac{e_{c, h}^{n+1}-e_{c, h}^{n}}{\Delta t}\right|_{c}^{2}+\frac{1}{2}\left|A_{c, h}^{1 / 2} \frac{e_{c, h}^{n+1}+e_{c, h}^{n}}{2}\right|_{c}^{2}
$$

where $I_{c, h}$ is the identity operator in $H_{c}$, and with

$$
\mathcal{E}_{f, h}^{n+1 / 2}=\frac{1}{2}\left|\mathcal{R}\left(\Delta t^{2} A_{f, h}\right)^{\frac{1}{2}} \frac{e_{f, h}^{n+1}-e_{f, h}^{n}}{\Delta t}\right|_{f}^{2}+\frac{1}{2}\left|A_{f, h}^{1 / 2} \frac{e_{f, h}^{n+1}+e_{f, h}^{n}}{2}\right|_{f}^{2}
$$

Proof. For the sake of conciseness, we only list here the main steps of the proof:

- compute the scalar product $(\cdot, \cdot)_{c}$ of the first equation of $(3.12)$ with

$$
\frac{e_{c, h}^{n+1}-e_{c, h}^{n-1}}{2 \Delta t},
$$

- compute the scalar product $(\cdot, \cdot)_{f}$ of the second equation of $(3.12)$ with

$$
\mathcal{P}_{p}\left(\Delta t^{2} A_{f, h}\right)^{-1} \frac{e_{f, h}^{n+1}-e_{f, h}^{n-1}}{2 \Delta t},
$$


- sum the two obtained equations and use the third equation of (3.12) to get rid of the term involving $\ell^{n}$.

- observe that $\mathcal{E}_{c, h}^{n+1 / 2}$ and $\mathcal{E}_{f, h}^{n+1 / 2}$ are positive quadratic energy functionals if Assumption 3.2 holds.

\subsubsection{Stability results.}

To obtain meaningful results we need more assumptions on how the spectral radius of $A_{f, h}$ behaves with respect to $h$ compare to the spectral radius of $A_{c, h}$. More precisely we assume the following property

Assumption 3.7. There exists $\beta$ independent of $h$ such that

$$
\frac{\rho_{f, h}}{\rho_{c, h}} \leq \beta^{2} .
$$

Let us now suppose that Assumption 3.2 holds. We introduce the positive scalar $C_{\mathcal{R}}$, independent of $h$, as

$$
C_{\mathcal{R}}:=\sup _{x \in\left[0,4 \alpha^{2} \beta^{2}\right]}|\mathcal{R}(x)|,
$$

where $\mathcal{R}(x)$ is given by (3.18). Since $\Delta t^{2} \rho_{f, h} \leq 4 \alpha^{2} \beta^{2}$ one can show that for all $v_{h}$ in $V_{f, h}$ the following inequality holds

$$
\left|\mathcal{R}\left(\Delta t^{2} A_{f, h}\right)^{\frac{1}{2}} v_{h}\right|_{f} \leq C_{\mathcal{R}}^{\frac{1}{2}}\left|v_{h}\right|_{f}
$$

Moreover we define $C_{\mathcal{Q}}$, independent of $h$, as

$$
C_{\mathcal{Q}}:=\sup _{x \in\left[0,4 \alpha^{2} \beta^{2}\right]}|\mathcal{Q}(x)|,
$$

where $\mathcal{Q}(x)$ is given by (3.19). Again, since $\Delta t^{2} \rho_{f, h} \leq 4 \alpha^{2} \beta^{2}$ one can show that for all $v_{h}$ in $V_{f, h}$ the following inequality holds

$$
\left|\mathcal{Q}\left(\Delta t^{2} A_{f, h}\right) v_{h}\right|_{f} \leq C_{\mathcal{Q}}\left|v_{h}\right|_{f}
$$

Theorem 3.8. Let Assumptions 2.2, 3.1, 3.2 and 3.7 hold. Then, there exists a scalar $C$ independent on $\mathcal{P}_{k}$, $\mathcal{P}_{p}, \Delta t$ and $h$ such that we have for $n \in\{1, \ldots, N\}$,

$$
\left(\mathcal{E}_{f, h}^{n-1 / 2}\right)^{\frac{1}{2}}+\left(\mathcal{E}_{c, h}^{n-1 / 2}\right)^{\frac{1}{2}} \leq C\left(1+C_{\mathcal{R}}^{\frac{1}{2}}+C_{\mathcal{Q}}\right)\left(\left(\mathcal{E}_{f, h}^{1 / 2}\right)^{\frac{1}{2}}+\left(\mathcal{E}_{c, h}^{1 / 2}\right)^{\frac{1}{2}}+\Delta t^{2}\right)
$$

Proof. In what follows the scalar $C$ - independent on $\mathcal{P}_{k}, \mathcal{P}_{p}, \Delta t$ and $h$ - is allowed to change from one line to the other. After summing equation (3.20) over $n=1$ to $n=N-1$ and taking into account equations (3.15) and (3.17), we find

$$
\mathcal{E}_{c, h}^{N-1 / 2}+\mathcal{E}_{f, h}^{N-1 / 2} \leq \mathcal{E}_{c, h}^{1 / 2}+\mathcal{E}_{f, h}^{1 / 2}+C \Delta t^{2}\left(\Xi_{c}^{N}+\Xi_{f}^{N}+\Pi_{f}^{N}+\Lambda_{f}^{N}\right)
$$

where

$$
\left\{\begin{array}{l}
\Xi_{c}^{N}=\Delta t \sum_{n=1}^{N-1}\left(\frac{\mathrm{d}^{4}}{\mathrm{~d} t^{4}} u_{c, h}\left(t^{n, \odot}\right), \frac{e_{c, h}^{n+1}-e_{c, h}^{n-1}}{2 \Delta t}\right)_{c}, \\
\Xi_{f}^{N}=\Delta t \sum_{n=1}^{N-1}\left(\mathcal{R}\left(\Delta t^{2} A_{f, h}\right) \frac{\mathrm{d}^{4}}{\mathrm{~d} t^{4}} u_{f, h}\left(t^{n, \boldsymbol{\bullet}}\right), \frac{e_{f, h}^{n+1}-e_{f, h}^{n-1}}{2 \Delta t}\right)_{f}, \\
\Pi_{f}^{N}=\Delta t \sum_{n=1}^{N-1}\left(A_{f, h} \frac{\mathrm{d}^{2}}{\mathrm{~d} t^{2}} u_{f, h}\left(t^{n, \boldsymbol{\bullet}}\right), \frac{e_{f, h}^{n+1}-e_{f, h}^{n-1}}{2 \Delta t}\right)_{f}, \\
\Lambda_{f}^{N}=\Delta t \sum_{n=1}^{N-1}\left(\mathcal{Q}\left(\Delta t^{2} A_{f, h}\right) A_{f, h} \frac{\mathrm{d}^{2}}{\mathrm{~d} t^{2}} u_{f, h}\left(t^{n}\right), \frac{e_{f, h}^{n+1}-e_{f, h}^{n-1}}{2 \Delta t}\right)_{f}
\end{array} .\right.
$$


The proof then proceeds in five steps. One step for the estimation of each of the four terms above and a final step that collects all the obtained estimations in order to obtain (3.26) using a discrete Gronwall's lemma.

Step 1. Estimation of $\Xi_{c}^{N}$. Following the proof given in [8] (proof 2.4 of Lem. 2.3 and appendix) it is possible to show that

$$
\left|\frac{e_{c, h}^{n+1}-e_{c, h}^{n-1}}{2 \Delta t}\right|_{c} \leq 2\left(\mathcal{E}_{c, h}^{n+1 / 2}\right)^{\frac{1}{2}}+2\left(\mathcal{E}_{c, h}^{n-1 / 2}\right)^{\frac{1}{2}} .
$$

It has to be noted that this inequality holds uniformly with respect to the time step (in the limit given by Assumption 3.2) and in particular it is valid if $\Delta t=2 / \sqrt{\rho_{c, h}}$. This result is not trivial: it is proven using a decomposition into low and high frequency components of the solution $u_{c, h}$. Then using Cauchy-Schwarz inequality and the estimate (3.27), as well as standard algebraic manipulations, one gets

$$
\begin{aligned}
\Xi_{c}^{N} & \leq \Delta t \sum_{n=1}^{N-1}\left|\frac{\mathrm{d}^{4}}{\mathrm{~d} t^{4}} u_{c, h}\left(t^{n,}\right)\right|_{c}\left|\frac{e_{c, h}^{n+1}-e_{c, h}^{n-1}}{2 \Delta t}\right|_{c} \\
& \leq C \sup _{t \in[0, T]}\left|\frac{\mathrm{d}^{4}}{\mathrm{~d} t^{4}} u_{c, h}(t)\right|_{c} \Delta t \sum_{n=0}^{N-1}\left(\mathcal{E}_{c, h}^{n+1 / 2}\right)^{\frac{1}{2}} .
\end{aligned}
$$

Using the stability estimate $(2.11)$ we obtain

$$
\Xi_{c}^{N} \leq C \Delta t \sum_{n=0}^{N-1}\left(\mathcal{E}_{c, h}^{n+1 / 2}\right)^{\frac{1}{2}} .
$$

Step 2. Estimation of $\Xi_{f}^{N}$. Writing $e_{f, h}^{n+1}-e_{f, h}^{n-1}=e_{f, h}^{n+1}-e_{f, h}^{n}+e_{f, h}^{n}-e_{f, h}^{n-1}$ and using the symmetry of $\mathcal{R}\left(\Delta t^{2} A_{f, h}\right)$ one can show, with the Cauchy-Schwarz and triangle inequalities, that

$$
\begin{aligned}
\Xi_{f}^{N} \leq & \frac{\Delta t}{2} \sum_{n=1}^{N-1}\left|\mathcal{R}\left(\Delta t^{2} A_{f, h}\right)^{\frac{1}{2}} \frac{\mathrm{d}^{4}}{\mathrm{~d} t^{4}} u_{f, h}\left(t^{n, \uparrow}\right)\right|_{f}\left(\left|\mathcal{R}\left(\Delta t^{2} A_{f, h}\right)^{\frac{1}{2}} \frac{e_{f, h}^{n+1}-e_{f, h}^{n}}{\Delta t}\right|_{f}\right. \\
& \left.+\left|\mathcal{R}\left(\Delta t^{2} A_{f, h}\right)^{\frac{1}{2}} \frac{e_{f, h}^{n}-e_{f, h}^{n-1}}{\Delta t}\right|_{f}\right)
\end{aligned}
$$

then, since by definition of the energy (3.22) we have

$$
\left|\mathcal{R}\left(\Delta t^{2} A_{f, h}\right)^{\frac{1}{2}} \frac{e_{f, h}^{n+1}-e_{f, h}^{n}}{\Delta t}\right|_{f} \leq \sqrt{2}\left(\mathcal{E}_{f, h}^{n+1 / 2}\right)^{\frac{1}{2}}
$$

we can simplify (3.31), and we obtain

$$
\begin{aligned}
\Xi_{f}^{N} & \leq \Delta t \frac{\sqrt{2}}{2} \sum_{n=1}^{N-1}\left|\mathcal{R}\left(\Delta t^{2} A_{f, h}\right)^{\frac{1}{2}} \frac{\mathrm{d}^{4}}{\mathrm{~d} t^{4}} u_{f, h}\left(t^{n, \uparrow}\right)\right|_{f}\left(\left(\mathcal{E}_{f, h}^{n+1 / 2}\right)^{\frac{1}{2}}+\left(\mathcal{E}_{f, h}^{n-1 / 2}\right)^{\frac{1}{2}}\right) \\
& \leq C C_{\mathcal{R}}^{\frac{1}{2}} \sup _{t \in[0, T]}\left|\frac{\mathrm{d}^{4}}{\mathrm{~d} t^{4}} u_{f, h}(t)\right|_{f} \Delta t \sum_{n=0}^{N-1}\left(\mathcal{E}_{f, h}^{n+1 / 2}\right)^{\frac{1}{2}} .
\end{aligned}
$$

Using the stability estimate (2.11) we obtain

$$
\Xi_{f}^{N} \leq C C_{\mathcal{R}}^{\frac{1}{2}} \Delta t \sum_{n=0}^{N-1}\left(\mathcal{E}_{f, h}^{n+1 / 2}\right)^{\frac{1}{2}} .
$$


Step 3. Estimation of $\Pi_{f}^{N}$. The difficulty here is that one can not expect in general to have a uniform bound on $A_{f, h} d_{t}^{2} u_{f, h}(t)$ in the norm in $H_{f}$. The standard strategy is to use the following equality

$$
\left(A_{f, h} u_{h}, v_{h}\right)_{q}=\left(A_{f, h}^{\frac{1}{2}} u_{h}, A_{f, h}^{\frac{1}{2}} v_{h}\right)_{q}, \quad \forall\left(u_{h}, v_{h}\right) \in V_{q, h} \times V_{q, h},
$$

then, a discrete by part integration in time. The objective is to "exchange space and time derivatives" between the error term and the solution to the semi-discrete problem. The by-part integration in time is done using the following algebraic rule: for all sequences of real numbers $\left\{v^{n}\right\}$ and $\left\{w^{n}\right\}$ we have

$$
\sum_{n=1}^{N-1} v^{n}\left(w^{n+1}-w^{n-1}\right)=-\sum_{n=1}^{N-2}\left(v^{n+1}-v^{n}\right)\left(w^{n+1}+w^{n}\right)+v^{N-1}\left(w^{N}+w^{N-1}\right)-v^{1}\left(w^{1}+w^{0}\right) .
$$

We apply the above equality to the term $\Pi_{f}^{N}$ and use property (3.34) as mentioned. We obtain

$$
\begin{aligned}
\Pi_{f}^{N}= & -\Delta t \sum_{n=1}^{N-2}\left(\frac{A_{f, h}^{\frac{1}{2}}}{\Delta t}\left(\frac{\mathrm{d}^{2}}{\mathrm{~d} t^{2}} u_{f, h}\left(t^{n+1, \boldsymbol{\wp}}\right)-\frac{\mathrm{d}^{2}}{\mathrm{~d} t^{2}} u_{f, h}\left(t^{n, \boldsymbol{\wp}}\right)\right), A_{f, h}^{\frac{1}{2}} \frac{e_{f, h}^{n+1}+e_{f, h}^{n}}{2}\right)_{f} \\
& +\left(A_{f, h}^{\frac{1}{2}} \frac{\mathrm{d}^{2}}{\mathrm{~d} t^{2}} u_{f, h}\left(t^{N-1, \boldsymbol{\wp}}\right), A_{f, h}^{\frac{1}{2}} \frac{e_{f, h}^{N}+e_{f, h}^{N-1}}{2}\right)_{f} \\
& -\left(A_{f, h}^{\frac{1}{2}} \frac{\mathrm{d}^{2}}{\mathrm{~d} t^{2}} u_{f, h}\left(t^{1, \boldsymbol{\kappa}}\right), A_{f, h}^{\frac{1}{2}} \frac{e_{f, h}^{1}+e_{f, h}^{0}}{2}\right)_{f}
\end{aligned}
$$

Moreover using the mean value theorem we find that

$$
\left|\frac{A_{f, h}^{\frac{1}{2}}}{\Delta t}\left(\frac{\mathrm{d}^{2}}{\mathrm{~d} t^{2}} u_{f, h}\left(t^{n+1, \boldsymbol{\infty}}\right)-\frac{\mathrm{d}^{2}}{\mathrm{~d} t^{2}} u_{f, h}\left(t^{n, \boldsymbol{\kappa}}\right)\right)\right|_{f} \leq \sup _{t \in[0, T]}\left|A_{f, h}^{\frac{1}{2}} \frac{\mathrm{d}^{3}}{\mathrm{~d} t^{3}} u_{f, h}(t)\right|_{f} \leq C,
$$

and by the definition of the energy (3.22) one gets

$$
\left|A_{f, h}^{\frac{1}{2}} \frac{e_{f, h}^{n+1}+e_{f, h}^{n}}{2}\right|_{f} \leq \sqrt{2}\left(\mathcal{E}_{f, h}^{n+1 / 2}\right)^{\frac{1}{2}}
$$

Injecting the estimate above as well as estimate (3.37) into (3.36), one obtains after using Cauchy-Schwarz inequality

$$
\Pi_{f}^{N} \leq C\left(\left(\mathcal{E}_{f, h}^{N-1 / 2}\right)^{\frac{1}{2}}+\left(\mathcal{E}_{f, h}^{1 / 2}\right)^{\frac{1}{2}}\right)+C \Delta t \sum_{n=1}^{N-1}\left(\mathcal{E}_{f, h}^{n+1 / 2}\right)^{\frac{1}{2}} .
$$

Step 4. Estimation of $\Lambda_{f}^{N}$. A similar strategy than for the estimation of $\Pi_{f}^{N}$ can be applied. For that it is essential to observe the following property

$$
\mathcal{Q}\left(\Delta t^{2} A_{f, h}\right) A_{f, h}=A_{f, h}^{\frac{1}{2}} \mathcal{Q}\left(\Delta t^{2} A_{f, h}\right) A_{f, h}^{\frac{1}{2}},
$$

that can be proven by diagonalisation of the operators involved on the family of eigenvectors of $A_{f, h}$. Then the same proof as in step 3 can be used. We obtain

$$
\Lambda_{f}^{N} \leq C C_{\mathcal{Q}}\left(\left(\mathcal{E}_{f, h}^{N-1 / 2}\right)^{\frac{1}{2}}+\left(\mathcal{E}_{f, h}^{1 / 2}\right)^{\frac{1}{2}}\right)+C C_{\mathcal{Q}} \Delta t \sum_{n=1}^{N-1}\left(\mathcal{E}_{f, h}^{n+1 / 2}\right)^{\frac{1}{2}}
$$

where $C_{\mathcal{Q}}$ is given by $(3.25)$. 
Step 5. Final energy estimate and Gronwall's lemma application. Combining inequalities (3.30), (3.33), (3.38) and (3.39) obtained above, we find

$$
\begin{aligned}
\mathcal{E}_{c, h}^{N-1 / 2}+\mathcal{E}_{f, h}^{N-1 / 2} \leq & C\left(\mathcal{E}_{c, h}^{1 / 2}+\mathcal{E}_{f, h}^{1 / 2}\right)+C \Delta t^{2}\left(1+C_{\mathcal{Q}}\right)\left(\left(\mathcal{E}_{f, h}^{N-1 / 2}\right)^{\frac{1}{2}}+\left(\mathcal{E}_{f, h}^{1 / 2}\right)^{\frac{1}{2}}\right) \\
& +C \Delta t^{3} \sum_{n=0}^{N-1}\left(\left(\mathcal{E}_{c, h}^{n+1 / 2}\right)^{\frac{1}{2}}+\left(1+C_{\mathcal{R}}^{\frac{1}{2}}+C_{\mathcal{Q}}\right)\left(\mathcal{E}_{f, h}^{n+1 / 2}\right)^{\frac{1}{2}}\right)
\end{aligned}
$$

Then using Young's inequality we write that

$$
C \Delta t^{2}\left(1+C_{\mathcal{Q}}\right)\left(\mathcal{E}_{f, h}^{m-1 / 2}\right)^{\frac{1}{2}} \leq \frac{C^{2} \Delta t^{4}}{2}\left(1+C_{\mathcal{Q}}\right)^{2}+\frac{1}{2} \mathcal{E}_{f, h}^{m-1 / 2},
$$

for $m=1$ and $m=N$, and, using the above estimation into (3.40) we obtain

$$
\mathcal{E}_{c, h}^{N-1 / 2}+\mathcal{E}_{f, h}^{N-1 / 2} \leq C\left(\mathcal{E}_{c, h}^{1 / 2}+\mathcal{E}_{f, h}^{1 / 2}\right)+C\left(1+C_{\mathcal{Q}}\right)^{2} \Delta t^{4}+C\left(1+C_{\mathcal{R}}^{\frac{1}{2}}+C_{\mathcal{Q}}\right) \Delta t^{3} \sum_{n=0}^{N-1}\left(\mathcal{E}_{c, h}^{n+1 / 2}+\mathcal{E}_{f, h}^{n+1 / 2}\right)^{\frac{1}{2}}
$$

To conclude let us use the following discrete Gronwall's lemma (see proof in Appendix B): for any real positive sequences $\left\{v^{n}\right\}$ and any positive scalar numbers $A$ and $B$ we have, for all $m \geq 1$,

$$
v^{m-1} \leq A+B \sum_{n=1}^{m-1}\left(v^{n}\right)^{\frac{1}{2}} \Rightarrow\left(v^{m-1}\right)^{\frac{1}{2}} \leq A^{\frac{1}{2}}+(m-1) B,
$$

where we use the convention that the sum $\sum\left(v^{n}\right)^{\frac{1}{2}}$ is equal to zero for $m=1$. Applying this result with $v^{n}=\mathcal{E}_{c, h}^{n+1 / 2}+\mathcal{E}_{f, h}^{n+1 / 2}$ in (3.41) we obtain, after some algebraic manipulations, the result of the theorem (note that we use the property $(N-1) \Delta t<T$ ).

Estimate (3.26) shows that it is important to obtain reasonable bounds on the coefficients $C_{\mathcal{R}}$ and $C_{\mathcal{Q}}$. In particular, if $\mathcal{P}(x)$ has some roots then these coefficients may blow up. This is the main difficulty that is addressed in Section 4.3.2 when constructing polynomials for explicit local time discretisation.

\subsubsection{Space-time convergence results.}

Corollary 3.9. If the assumptions of Theorems 2.3 and 3.8 hold, then, there exists $C$ independent of $\Delta t$ and $h$ such that, for all $n \in\{1, \ldots, N-1\}$

$$
\left\|\left\{\boldsymbol{u}_{h}^{n}\right\}_{1 / 4}-\boldsymbol{u}\left(t^{n}\right)\right\| \leq C\left(\Delta t^{2}+\delta_{h}\right) .
$$

Proof. In the proof the notation $C$ refers to a positive scalar independent of $\Delta t$ and $h$ than can change from one line to another. Our first objective is to estimate

$$
\left|\frac{\boldsymbol{e}_{h}^{n+1}-\boldsymbol{e}_{h}^{n-1}}{2 \Delta t}\right|
$$

by the energy terms. To do so we use the theory developed in [8] that relies on a separation of the unknowns into two orthogonal subspaces spanned by the eigenvectors of the matrices $A_{c, h}$ and $A_{f, h}$. It is then shown in [8] that (3.27) holds, i.e,

$$
\left|\frac{e_{c, h}^{n+1}-e_{c, h}^{n-1}}{2 \Delta t}\right|_{c} \leq 2\left(\left(\mathcal{E}_{c, h}^{n+1 / 2}\right)^{\frac{1}{2}}+\left(\mathcal{E}_{c, h}^{n-1 / 2}\right)^{\frac{1}{2}}\right)
$$


To show that a similar identity holds for $\left(e_{f, h}^{n+1}-e_{f, h}^{n-1}\right) / 2 \Delta t$, we check the hypothesis 2.2 of [8]. More precisely, since $\mathcal{R}(0)=1$ one can check that there exists $\eta>0, C_{\mathcal{K}}>0$ and $C_{\mathcal{P}}>0$ such that

$$
C_{\mathcal{K}} \leq \mathcal{R}(x), \quad \forall x \in[0, \eta], \quad C_{\mathcal{P}} \leq x \quad \forall x \in\left(\eta, 4 \alpha^{2} \beta^{2}\right]
$$

then, following the proof of Lemma 2.3 in [8] we obtain

$$
\left|\frac{e_{f, h}^{n+1}-e_{f, h}^{n-1}}{2 \Delta t}\right|_{f} \leq\left(C_{\mathcal{P}}^{-1 / 2}+\frac{C_{\mathcal{K}}^{-1 / 2}}{2}\right) \sqrt{2}\left(\left(\mathcal{E}_{f, h}^{n+1 / 2}\right)^{\frac{1}{2}}+\left(\mathcal{E}_{f, h}^{n-1 / 2}\right)^{\frac{1}{2}}\right)
$$

Combining the two estimates above we obtain,

$$
\left|\frac{\boldsymbol{e}_{h}^{n+1}-\boldsymbol{e}_{h}^{n-1}}{2 \Delta t}\right| \leq C\left(\left(\mathcal{E}_{h}^{n+1 / 2}\right)^{\frac{1}{2}}+\left(\mathcal{E}_{h}^{n-1 / 2}\right)^{\frac{1}{2}}\right), \quad\left(\mathcal{E}_{h}^{n+1 / 2}\right)^{\frac{1}{2}}=\left(\mathcal{E}_{f, h}^{n+1 / 2}\right)^{\frac{1}{2}}+\left(\mathcal{E}_{c, h}^{n+1 / 2}\right)^{\frac{1}{2}} .
$$

From this inequality we deduce straightforwardly that

$$
\left|\frac{\boldsymbol{e}_{h}^{n+1}+\boldsymbol{e}_{h}^{n}}{2}\right| \leq\left|\frac{\boldsymbol{e}_{h}^{1}+\boldsymbol{e}_{h}^{0}}{2}\right|+C \Delta t \sum_{m=0}^{n}\left(\mathcal{E}_{h}^{m+1 / 2}\right)^{\frac{1}{2}}, \quad \forall n \in\{1, \cdots, N-1\} .
$$

Observe now, thanks to the coercivity $(2.2)$ of the bilinear forms $a(\cdot, \cdot)$, that for all $n \in\{1, \ldots, N-1\}$,

$$
\left\|\frac{\boldsymbol{e}_{h}^{n+1}+\boldsymbol{e}_{h}^{n}}{2}\right\| \leq C\left|\frac{\boldsymbol{e}_{h}^{n+1}+\boldsymbol{e}_{h}^{n}}{2}\right|+C \mathcal{E}_{h}^{n+1 / 2} \text { and }\left\|\left\{\boldsymbol{e}_{h}^{n}\right\}_{1 / 4}\right\| \leq\left\|\frac{\boldsymbol{e}_{h}^{n+1}+\boldsymbol{e}_{h}^{n}}{2}\right\|+\left\|\frac{e_{h}^{n}+\boldsymbol{e}_{h}^{n-1}}{2}\right\| .
$$

We obtain

$$
\left\|\left\{\boldsymbol{e}_{h}^{n}\right\}_{1 / 4}\right\| \leq C\left|\frac{\boldsymbol{e}_{h}^{1}+\boldsymbol{e}_{h}^{0}}{2}\right|+C \sup _{n \in\{0, \cdots, N-1\}} \mathcal{E}_{h}^{n+1 / 2}+C \Delta t \sum_{m=0}^{N-1}\left(\mathcal{E}_{h}^{m+1 / 2}\right)^{\frac{1}{2}} .
$$

We now study the initial terms $\boldsymbol{e}_{h}^{0}$ and $\boldsymbol{e}_{h}^{1}$. By definition we have

$$
\boldsymbol{e}_{h}^{0}=\mathbf{0}, \quad \boldsymbol{e}_{h}^{1}=\boldsymbol{u}_{h}(\Delta t)-\boldsymbol{u}_{h}^{1}=\frac{\Delta t^{3}}{6} \frac{\mathrm{d}^{3}}{\mathrm{~d} t^{3}} \boldsymbol{u}_{h}\left(t^{\star}\right)
$$

where $0 \leq t \leq \Delta t$. Thanks to Assumption 2.2 we have a uniform bound of $\boldsymbol{u}_{h}$ in $C^{3}\left([0, T], \boldsymbol{V}_{h}\right)$ for the supremum norm in time and $\|\cdot\|$ in space, moreover we have

$$
\mathcal{E}_{h}^{1 / 2} \leq C\left|\frac{\boldsymbol{e}_{h}^{1}-\boldsymbol{e}_{h}^{0}}{\Delta t}\right|+\left\|\frac{\boldsymbol{e}_{h}^{1}+\boldsymbol{e}_{h}^{0}}{2}\right\| \quad \text { hence } \quad \mathcal{E}_{h}^{1 / 2} \leq C \Delta t^{2} .
$$

Then, estimation (3.44) can be simplified using Theorem 3.8 and the above estimation of $\mathcal{E}_{h}^{1 / 2}$. We obtain

$$
\left\|\left\{\boldsymbol{e}_{h}^{n}\right\}_{1 / 4}\right\| \leq C\left(\Delta t^{2}+C \sup _{n \in\{0, \cdots, N-1\}} \mathcal{E}_{h}^{n+1 / 2}\right) \Rightarrow\left\|\left\{\boldsymbol{e}_{h}^{n}\right\}_{1 / 4}\right\| \leq C \Delta t^{2} .
$$

The statement of the corollary is obtained using an adequate decomposition of the difference $\left\{\boldsymbol{u}_{h}^{n}\right\}_{1 / 4}-u\left(t^{n}\right)$ and triangle inequalities. More precisely we have

$$
\left\|\left\{\boldsymbol{u}_{h}^{n}\right\}_{1 / 4}-\boldsymbol{u}\left(t^{n}\right)\right\| \leq\left\|\left\{\boldsymbol{e}_{h}^{n}\right\}_{1 / 4}\right\|+\left\|\left\{\boldsymbol{u}_{h}\left(t^{n}\right)\right\}_{1 / 4}-\boldsymbol{u}_{h}\left(t^{n}\right)\right\|+\left\|\boldsymbol{e}_{h}\left(t^{n}\right)\right\|,
$$

where the first term can be estimated by (3.45); the second term is uniformly bounded (with respect to $h$ ) by $\Delta t^{2}$ since $\boldsymbol{u}_{h} \in C^{2}\left([0, T], \boldsymbol{V}_{h}\right)$ and (2.11) holds; and the last term can be estimated using Theorem 2.3. 
Corollary 3.9 means that the "good" quantity that approximates $\boldsymbol{u}\left(t^{n}\right)$ is $\widetilde{\boldsymbol{u}}_{h}^{n}=\left\{\boldsymbol{u}_{h}^{n}\right\}_{1 / 4}$. One way to obtain this quantity is by post-processing the obtained solution, a more efficient approach is to compute it directly, indeed, by linearity $\widetilde{\boldsymbol{u}}_{h}^{n}$ can be computed solving (3.1) for $n \in\{1, \ldots, N-2\}$ with source term $\widetilde{\boldsymbol{f}}_{h}^{n}=\left\{\boldsymbol{f}_{h}\left(t^{n}\right)\right\}_{1 / 4}$ instead of $\boldsymbol{f}_{h}\left(t^{n}\right)$ and with initial data

$$
\widetilde{\boldsymbol{u}}_{h}^{1}=\frac{\boldsymbol{u}_{h}^{0}+2 \boldsymbol{u}_{h}^{1}+\boldsymbol{u}_{h}^{2}}{4}, \quad \widetilde{\boldsymbol{u}}_{h}^{2}=\frac{\boldsymbol{u}_{h}^{1}+2 \boldsymbol{u}_{h}^{2}+\boldsymbol{u}_{h}^{3}}{4} .
$$

This involves only a small change in the computation of the source terms and the initial data in (3.1) but allows to recover the expected estimate $\left\|\widetilde{\boldsymbol{u}}_{h}^{n}-\boldsymbol{u}\left(t^{n}\right)\right\|=O\left(\Delta t^{2}+\delta_{h}\right)$.

\section{DeRIVATION OF LOCAL IMPLiCIT OR EXPLiCIT TIME DISCRETISATIONS}

In this section we derive three specific local time discretisations that enter the framework described in Section 3. The presented schemes are of increasing complexity and are constructed assuming $\rho_{c, h}$ and $\rho_{f, h}$ known.

\subsection{Local implicit scheme}

Local implicit strategies for wave equations have been developed and analysed by several authors, see for instance $[18,21,30,39]$. Moreover in [7] a second order and a fourth order local implicit time discretisation adapted to domain decomposition have been constructed. The second order method of [7] fits naturally into the family of discrete problems (3.1) that we have constructed. It is obtained by choosing

$$
\mathcal{P}_{k}(x)=1 \quad \text { and } \quad \mathcal{P}_{p}(x)=1 .
$$

With this choice, Assumption 3.2 holds for any $0<\alpha \leq 1$. Moreover we have

$$
\mathcal{Q}(x) \equiv 0 \quad \text { and } \quad \mathcal{R}(x) \equiv 1 .
$$

The complete scheme reads

$$
\begin{cases}\frac{u_{c, h}^{n+1}-2 u_{c, h}^{n}+u_{c, h}^{n-1}}{\Delta t^{2}}+A_{c, h} u_{c, h}^{n}+B_{c, h}^{t} \lambda_{h}^{n}=f_{c, h}\left(t^{n}\right) & \text { in } V_{c, h}, \\ \frac{u_{f, h}^{n+1}-2 u_{f, h}^{n}+u_{f, h}^{n-1}}{\Delta t^{2}}+A_{f, h}\left\{u_{f, h}^{n}\right\}_{1 / 4}-B_{f, h}^{t} \lambda_{h}^{n}=f_{f, h}\left(t^{n}\right) & \text { in } V_{f, h}, \\ B_{c, h} u_{c, h}^{n}=B_{f, h} u_{f, h}^{n} & \text { in } L_{h} .\end{cases}
$$

Notice that this means that the first equation of (2.8) is discretised with an explicit leap-frog scheme, while the second is discretised with an unconditionally stable implicit $\theta$-scheme with $\theta=1 / 4$. It has been shown in [7] that at each time iteration, one needs to solve the following problem

$$
\left(\begin{array}{cc}
\frac{1}{\Delta t^{2}} I_{f, h}+\frac{1}{4} A_{f, h} & -B_{f, h}^{t} \\
B_{f, h} & B_{c, h}^{t} B_{c, h}
\end{array}\right)\left(\begin{array}{c}
u_{f, h}^{n+1} \\
\lambda_{h}^{n}
\end{array}\right)=\left(\begin{array}{c}
\hat{f}_{f, h}^{n} \\
\hat{g}_{h}^{n}
\end{array}\right),
$$

where $I_{f, h}$ is the identity operator in $V_{f, h}$ and where $\hat{f}_{h}^{n}$ and $\hat{g}_{h}^{n}$ are some source terms that depend on previous iterates and of $\boldsymbol{f}_{h}\left(t^{n}\right)$. The invertibility of the above system is guaranteed if the discrete inf-sup condition (2.10) holds as explained in Section 3.1. Since $\mathcal{Q}(x)$ and $\mathcal{R}(x)$ are independent of $x$ then $C_{\mathcal{Q}}=0$ and $C_{\mathcal{R}}=1$ are obviously independent of $\beta$ that can be arbitrarily high (hence the ratio $\rho_{f, h} / \rho_{c, h}$ can be arbitrarily high). Finally, the application of Corollary 3.9 proves the space-time convergence of (4.1). 
Note that, when applied to the wave equation (2.1), solving System (4.2) corresponds to solving the wave equation in $\Omega_{f}$ with an implicit scheme augmented by some operator acting on boundaries that accounts for the transmission of fluxes between $\Omega_{c}$ and $\Omega_{f}$ as well as the equality between $u_{c}$ and $u_{f}$ on $\Sigma$. This scheme is particularly adapted if a very strong and very local heterogeneity is considered in the propagating medium. In that case (4.2) is not well conditioned but the algebraic system has a small size and can be solved efficiently.

\subsection{Stabilised explicit scheme}

Our objective in this section is to construct a time discretisation that allows to treat situations for which we have

$$
\rho_{f, h} \leq 4 \rho_{c, h},
$$

that is to say $\beta=2$ in Assumption 3.7. Note that we expect $\rho_{f, h} \simeq 4 \rho_{c, h}$ for the scheme to be meaningful and efficient. For instance, in the case of standard $\mathbb{P}_{k}$-finite elements on a uniform mesh for the scalar wave equation (2.1), if the mesh size used to discretise $\Omega_{f}$ is two times smaller than the mesh size used to discretise $\Omega_{c}$, we have $\rho_{f, h}=4 \rho_{c, h}$.

The scheme is constructed by choosing

$$
\mathcal{P}_{p}(x)=1-\frac{x}{16}
$$

and setting

$$
\mathcal{P}_{k}(x)=1-x \frac{\mathcal{P}_{p}(x)}{4}=1-\frac{x}{4}+\frac{x^{2}}{64} \quad\left(=\left(\frac{x}{8}-1\right)^{2}\right) .
$$

With this choice, scheme (3.1) is explicit (see Rem. 3.3). The complete scheme reads

$$
\left\{\begin{aligned}
\frac{u_{c, h}^{n+1}-2 u_{c, h}^{n}+u_{c, h}^{n-1}}{\Delta t^{2}}+A_{c, h} u_{c, h}^{n}+B_{c, h}^{t} \lambda_{h}^{n}=f_{c, h}\left(t^{n}\right) & \text { in } V_{c, h}, \\
\frac{u_{f, h}^{n+1}-2 u_{f, h}^{n}+u_{f, h}^{n-1}}{\Delta t^{2}} & \\
\quad+\left(I_{f, h}-\frac{\Delta t^{2}}{16} A_{f, h}\right)\left(A_{f, h} u_{f, h}^{n}-B_{f, h}^{t} \lambda_{h}^{n}-f_{f, h}\left(t^{n}\right)\right)=0 & \text { in } V_{f, h}, \\
B_{c, h} u_{c, h}^{n}=B_{f, h} u_{f, h}^{n} & \text { in } L_{h} .
\end{aligned}\right.
$$

Observe that at each time iteration, computing $\lambda_{h}^{n}$ requires to solve:

$$
\begin{aligned}
\left(B_{c, h} B_{c, h}^{t}+B_{f, h}\left(I_{f, h}-\frac{\Delta t^{2}}{16} A_{f, h}\right) B_{f, h}^{t}\right) \lambda_{h}^{n}= & B_{c, h} f_{c, h}\left(t^{n}\right)-B_{c, h} A_{c, h} u_{c, h}^{n} \\
& +B_{f, h}\left(I_{f, h}-\frac{\Delta t^{2}}{16} A_{f, h}\right)\left(A_{f, h} u_{f, h}^{n}-f_{f, h}\left(t^{n}\right)\right) .
\end{aligned}
$$

Then $\lambda_{h}^{n}$ is used to compute $u_{c, h}^{n+1}$ and $u_{f, h}^{n+1}$ explicitly using the first two equations of (4.6). The well-posedness property of (4.7) is a consequence of the discrete inf-sup condition (2.10). To apply Corollary 3.9 one needs to check that Assumption 3.2 holds. Since we have assumed $\beta=2$ (i.e. $\rho_{f, h} \leq 4 \rho_{c, h}$ ), we need to check (3.4), which reads, using (3.5),

$$
\mathcal{P}_{k}(x) \geq 0, \quad \mathcal{P}_{p}(x)>0, \quad \forall x \in\left[0,16 \alpha^{2}\right] .
$$

From the definition of $\mathcal{P}_{k}(x)$ given by (4.5) (see Fig. 1) it is clear that $\mathcal{P}_{k}(x) \geq 0$ for all positive $\alpha \leq 1$ (it has a double root at $x=8)$. However from the definition of (4.4) we see that $\mathcal{P}_{p}(x)>0$ only if $\alpha$ is strictly less than one, moreover we have

$$
\mathcal{Q}(x)=\frac{-\frac{3}{16}+\frac{x}{64}}{1-\frac{x}{16}}, \quad \mathcal{R}(x)=\frac{1-\frac{x}{4}+\frac{x^{2}}{64}}{1-\frac{x}{16}} .
$$




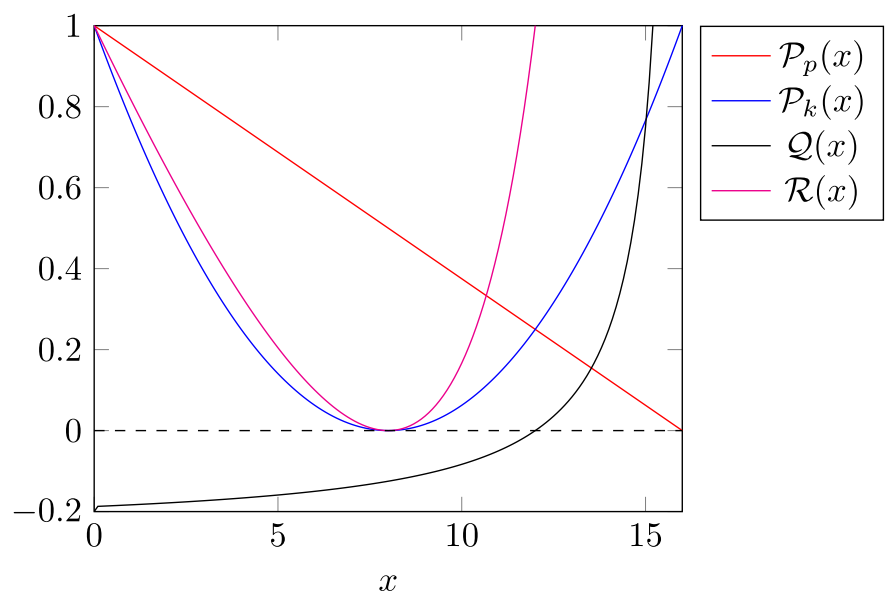

FiguRE 1. Representation of $\mathcal{P}_{p}(x)$ defined by (4.4), $\mathcal{P}_{k}(x)$ defined by $(4.5)$ and $\mathcal{Q}(x)$ and $\mathcal{R}(x)$ given by (4.8).

and therefore

$$
C_{\mathcal{Q}}=\max _{x \in\left[0,16 \alpha^{2}\right]}|\mathcal{Q}(x)| \underset{\alpha \rightarrow 1}{\sim} \frac{1}{16\left(1-\alpha^{2}\right)}, \quad C_{\mathcal{R}}=\max _{x \in\left[0,16 \alpha^{2}\right]}|\mathcal{R}(x)| \underset{\alpha \rightarrow 1}{\sim} \frac{1}{\left(1-\alpha^{2}\right)} .
$$

This estimate illustrates that the value $\alpha=1$ is forbidden to apply Theorem 3.8. However we will see that in practice a value really close to 1 gives satisfactory results (to back up this claim, several space-time convergence curves for different values of $\alpha$ are presented in Sect. 5.2). To conclude, we have constructed a time discretisation that is stable and convergent if $\rho_{f, h} \leq 4 \rho_{c, h}$ and $\Delta t$ is chosen strictly below the optimal value $2 / \sqrt{\rho_{c, h}}$.

\subsection{Local time discretisation using the Leap-Frog Chebychev method}

\subsubsection{Principle}

In the same spirit than Section 4.2, we construct now a method that can be characterised as a conservative local time stepping technique with an implicit treatment of transmission terms. As in Section 4.2, the unknown $u_{f, h}^{n}$ should be explicitly updated, hence following Remark 3.3, we assume that $\mathcal{P}_{k}(x)$ satisfies

$$
\mathcal{P}_{k}(x)=1-x \frac{\mathcal{P}_{p}(x)}{4} .
$$

The complete scheme reads

$$
\begin{cases}\frac{u_{c, h}^{n+1}-2 u_{c, h}^{n}+u_{c, h}^{n-1}}{\Delta t^{2}}+A_{c, h} u_{c, h}^{n}+B_{c, h}^{t} \lambda_{h}^{n}=f_{c, h}\left(t^{n}\right) & \text { in } V_{c, h}, \\ \frac{u_{f, h}^{n+1}-2 u_{f, h}^{n}+u_{f, h}^{n-1}}{\Delta t^{2}}+\mathcal{P}_{p}\left(\Delta t^{2} A_{f, h}\right)\left(A_{f, h} u_{f, h}^{n}-B_{f, h}^{t} \lambda_{h}^{n}-f_{f, h}\left(t^{n}\right)\right)=0 & \text { in } V_{f, h}, \\ B_{c, h} u_{c, h}^{n}=B_{f, h} u_{f, h}^{n} & \text { in } L_{h} .\end{cases}
$$

We do not yet specify the polynomial $\mathcal{P}_{p}(x)$, but from Assumption 3.1 (consistency assumption) we must have $\mathcal{P}_{p}(0)=\mathcal{P}_{k}(0)=1$. Notice that by definition (4.9) we have

$$
\mathcal{P}_{p}(0)=1 \quad \Rightarrow \quad \mathcal{P}_{k}(0)=1
$$


Our objective is then to construct a sequence of functions $\mathcal{P}_{p, \ell}$ that satisfy the properties

$$
\begin{cases}\mathcal{P}_{p, \ell} \text { is a polynomial } & \\ \mathcal{P}_{p, \ell}(0)=1, & \forall x \in\left[0,4 \beta_{\ell}^{2}\right] \\ 1-x \frac{\mathcal{P}_{p, \ell}(x)}{4} \geq 0, & \forall x \in\left[0,4 \beta_{\ell}^{2}\right] \\ \mathcal{P}_{p, \ell}(x)>0, & \end{cases}
$$

for a monotonically increasing sequence $\beta_{\ell}>2$. To satisfy Assumption 3.2 (stability assumption) it is sufficient to check that

$$
\Delta t^{2} \rho_{c, h}=4 \alpha^{2} \quad \alpha \in[0,1] \text { and } \Delta t^{2} \rho_{f, h} \leq 4 \beta_{\ell}^{2} \quad\left(\underset{(3.5)}{\Rightarrow} \quad \frac{\rho_{f, h}}{\rho_{c, h}} \leq \frac{\beta_{\ell}^{2}}{\alpha^{2}}\right)
$$

The last inequality means that Assumption 3.7 is verified with $\beta=\beta_{\ell} / \alpha$. Observe that this is an improvement compared to condition (4.3) associated to scheme (4.6).

In Section 4.3.2 below we present a procedure to construct the sequence of polynomials that satisfy the property (4.11) for some increasing sequence of $\beta_{\ell}$. In Section 4.3.3 we apply the algorithm of Section 4.3.2 below and construct a family of polynomials for which we have

$$
\beta_{2} \simeq 3, \beta_{3} \simeq 4 \text { and } \beta_{4} \simeq 5
$$

Remark 4.1. The stability condition of the scheme (4.10) with $\mathcal{P}_{p} \equiv \mathcal{P}_{p, \ell}$ can be rewritten

$$
\Delta t^{2} \leq \min \left(\frac{4}{\rho_{c, h}}, \frac{4 \beta_{\ell}^{2}}{\rho_{f, h}}\right) .
$$

Because of (4.12), this is clearly in improvement compared to the stability condition (3.10).

\subsubsection{Construction of a parametrized polynomials sequence}

To construct the sequence of polynomials that satisfy property (4.11) for a monotonically increasing sequence $\beta_{\ell}$, we start from the polynomials introduced in [24] that correspond to shifted and stretched Chebychev's polynomials. They are given by

$$
\widetilde{\mathcal{P}}_{p, \ell}(x)=\frac{2}{x}\left[1-\mathcal{T}_{\ell+1}\left(1-\frac{2 x}{4(\ell+1)^{2}}\right)\right]
$$

where $\mathcal{T}_{\ell}(x)$ is the $\ell$ th Chebychev polynomial. The first polynomials are given by

$$
\mathcal{T}_{3}(x)=4 x^{3}-3 x, \quad \mathcal{T}_{4}(x)=8 x^{4}-8 x^{2}+1, \quad \mathcal{T}_{5}(x)=16 x^{5}-20 x^{3}+5 x,
$$

hence for $\ell=\{2,3,4\}$, we have

$$
\begin{aligned}
& \widetilde{\mathcal{P}}_{p, 2}(x)=1-\frac{6}{3^{4}} x+\frac{1}{3^{6}} x^{2}, \quad \widetilde{\mathcal{P}}_{p, 3}(x)=1-\frac{20}{4^{4}} x+\frac{8}{4^{6}} x^{2}-\frac{1}{4^{8}} x^{3}, \\
& \widetilde{\mathcal{P}}_{p, 4}(x)=1-\frac{50}{5^{4}} x+\frac{35}{5^{6}} x^{2}-\frac{10}{5^{8}} x^{3}+\frac{1}{5^{10}} x^{4} .
\end{aligned}
$$

It is proven in [24] that the polynomials $\widetilde{\mathcal{P}}_{p, \ell}(x)$ satisfy

$$
\widetilde{\mathcal{P}}_{p, \ell}(0)=1, \quad 1-\frac{x \widetilde{\mathcal{P}}_{p, \ell}(x)}{4} \geq 0, \quad \widetilde{\mathcal{P}}_{p, \ell}(x) \geq 0 \quad \forall x \in\left[0,4(\ell+1)^{2}\right] .
$$




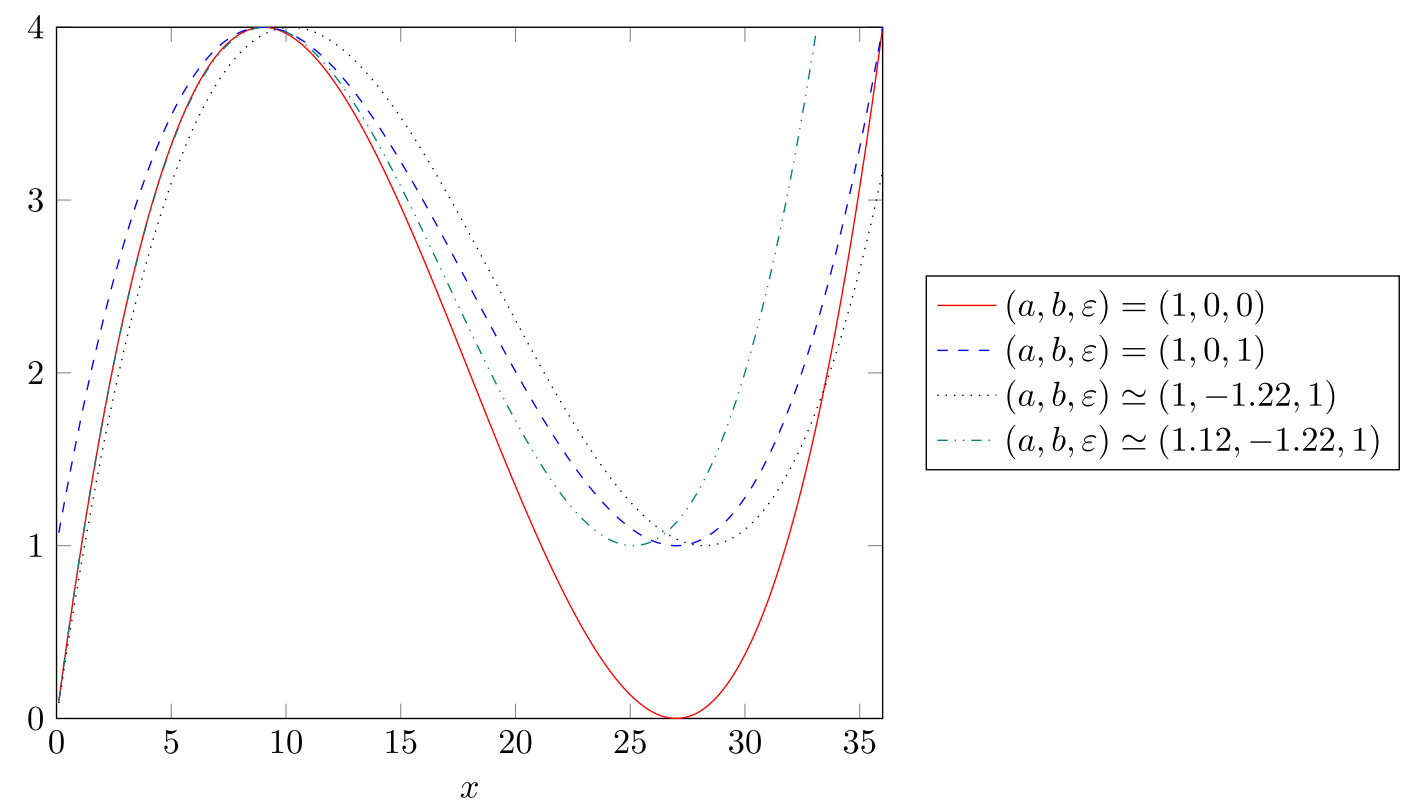

FiguRE 2. Representation of the polynomial $x \mathcal{P}_{p, \ell}^{\varepsilon}(x)$ given by (4.16) for $\ell=2$ and for different values of the parameters $(a, b, \varepsilon)$ obtained by following the procedure of paragraph 4.3.2.

The polynomials satisfy the good requirements that we have stated in order to construct the local time stepping process, i.e. (4.11), except for the fact that the $\widetilde{\mathcal{P}}_{p, \ell}(x)$ do vanish for some $x \leq 4(\ell+1)^{2}$. An idea used in [40] and [32] in the context of stabilisation of the Runge-Kutta method is to transform $\widetilde{\mathcal{P}}_{p, \ell}(x)$ to obtain the required behavior (i.e. $\widetilde{\mathcal{P}}_{p, \ell}(x)>0$ ). Note that a similar idea is used concurrently in the context of non-linear wave propagation phenomena in [15]. We define the family of functions $\mathcal{P}_{p, \ell}^{\varepsilon}(x)$ parametrized by $(a, b, \varepsilon)$ such that, for $\varepsilon$ positive and sufficiently small,

$$
\mathcal{P}_{p, \ell}^{\varepsilon}(x)=\frac{1}{x}\left(\left(1-\frac{\varepsilon}{4}\right)(a x+b) \widetilde{\mathcal{P}}_{p, \ell}(a x+b)+\varepsilon\right),
$$

see Figure 2 for an illustration of a representation of the polynomial $\mathcal{P}_{p, \ell}^{\varepsilon}$ for various parameters values. Note that if $a=1, b=0$ and $\varepsilon=0$ one recovers $\mathcal{P}_{p, \ell}^{\varepsilon}=\widetilde{\mathcal{P}}_{p, \ell}$.

Let us suppose given $0<\varepsilon<4$ small enough. We propose a procedure (see again Fig. 2) that computes $a \equiv a^{\varepsilon}$ and $b \equiv b^{\varepsilon}$ such that $\mathcal{P}_{p, \ell}^{\varepsilon}$ is a well-defined polynomial and consistency as well as stability are ensured. Namely, one should check that equations (4.11) holds.

Step i. From the definition (4.16) one can check that $\mathcal{P}_{p, \ell}^{\varepsilon}$ is a polynomial if the a-priori blow-up at $x=0$ is compensated, this means, that one should have,

$$
\left(1-\frac{\varepsilon}{4}\right) b^{\varepsilon} \widetilde{\mathcal{P}}_{p, \ell}\left(b^{\varepsilon}\right)+\varepsilon=0 \Rightarrow b^{\varepsilon} \widetilde{\mathcal{P}}_{p, \ell}\left(b^{\varepsilon}\right)=-\frac{\varepsilon}{1-\frac{\varepsilon}{4}} .
$$

It can be observed that $b^{\varepsilon}$ is a root of polynomial of order $\ell+1$. However since the polynomial $x \widetilde{\mathcal{P}}_{p, \ell}(x)$ behave like the linear function $x$ is a neighborhood of $x=0$ for $\varepsilon$ positive and sufficiently small there exists a real negative solution to (4.17). Hence, we choose $b^{\varepsilon}$ as the negative solution to (4.17) with the smallest absolute value. Note that we have $b^{\varepsilon} \rightarrow 0$ when $\varepsilon \rightarrow 0$. 
TABLE 1. Computed values of $a$ and $b$ for given values of $\varepsilon$ and associated $\beta_{\ell}^{\varepsilon}$.

\begin{tabular}{lllll}
\hline \hline$\ell$ & $\varepsilon$ & $b^{\varepsilon}$ & $a^{\varepsilon}$ & $\beta_{\ell}^{\varepsilon}$ \\
\hline 2 & 1 & -1.220497601922388 & 1.123332443935161 & 2.878 \\
2 & 0.5 & -0.548885078878804 & 1.055702443069509 & 2.941 \\
2 & 0.1 & -0.101795082372209 & 1.010360937184039 & 2.988 \\
\hline 3 & 1 & -1.214605698792632 & 1.112468647367209 & 3.828 \\
3 & 0.5 & -0.547676655750322 & 1.051055803796928 & 3.918 \\
3 & 0.1 & -0.101753177452728 & 1.009529277032937 & 3.984 \\
\hline 4 & 1 & -1.211812534393700 & 1.107473444638217 & 4.779 \\
4 & 0.5 & -0.547112834621174 & 1.048910062073242 & 4.895 \\
4 & 0.1 & -0.101733760636154 & 1.009144480323238 & 4.979 \\
\hline
\end{tabular}

Step ii. To satisfy Assumption 3.1, i.e. the consistency assumption, one must check that $\mathcal{P}_{p, \ell}^{\varepsilon}(0)=1$. To do so we first differentiate (4.16) with respect to $x$, we obtain

$$
\mathcal{P}_{p, \ell}^{\varepsilon}(x)+x\left(\mathcal{P}_{p, \ell}^{\varepsilon}\right)^{\prime}(x)=\left(1-\frac{\varepsilon}{4}\right) a^{\varepsilon}\left(\widetilde{\mathcal{P}}_{p, \ell}\left(a^{\varepsilon} x+b^{\varepsilon}\right)+\left(a^{\varepsilon} x+b^{\varepsilon}\right) \widetilde{\mathcal{P}}_{p, \ell}^{\prime}\left(a^{\varepsilon} x+b^{\varepsilon}\right)\right)
$$

and therefore

$$
\mathcal{P}_{p, \ell}^{\varepsilon}(0)=1 \quad \Rightarrow \quad a^{\varepsilon}=\frac{1}{\left(1-\frac{\varepsilon}{4}\right)\left(\widetilde{\mathcal{P}}_{p, \ell}\left(b^{\varepsilon}\right)+b^{\varepsilon} \widetilde{\mathcal{P}}_{p, \ell}^{\prime}\left(b^{\varepsilon}\right)\right)} .
$$

Note that for $\varepsilon$ positive and small enough the coefficient $a^{\varepsilon}$ is well defined and close to one and we we have $a^{\varepsilon} \rightarrow 1$ when $\varepsilon \rightarrow 0$.

Step iii. The last step concerns the definition of $\beta_{\ell}^{\varepsilon}$ such that (4.11c) and (4.11d) hold. From the definition (4.16) and the property (4.15) one can see that (4.11) holds for

$$
4\left(\beta_{\ell}^{\varepsilon}\right)^{2}=\frac{4(\ell+1)^{2}-b^{\varepsilon}}{a^{\varepsilon}} .
$$

Notice that $\beta_{\ell}^{\varepsilon} \rightarrow \ell+1$ when $\varepsilon \rightarrow 0$. Regarding stability, the resulting scheme indeed needs the constants $C_{\mathcal{R}}$ and $C_{\mathcal{Q}}$ to be bounded in order to provide second order accuracy in the $\|\cdot\|_{q}$ norm. If $\mathcal{P}_{p, \ell}^{\varepsilon}$ reaches 0 , $C_{\mathcal{R}}$ and $C_{\mathcal{Q}}$ degenerate. For the value of $\beta_{\ell}^{\varepsilon}$ given above, it can be shown that $4\left(\beta_{\ell}^{\varepsilon}\right)^{2} \mathcal{P}_{p, \ell}^{\varepsilon}\left(4\left(\beta_{\ell}^{\varepsilon}\right)^{2}\right)=\{\varepsilon, 4\}$ depending on the parity of $p$.

\subsubsection{Numerical construction}

In this subsection we apply the algorithm explained above for $\ell=2$ to $\ell=4$. Since the proposed algorithm is parametrized by $\varepsilon$ we choose $\varepsilon \in\{0.1,0.5,1\}$. Table 1 gives the values of $\left(a^{\varepsilon}, b^{\varepsilon}\right)$ that are computed, along with the corresponding values of $\beta_{\ell}^{\varepsilon}$. The script used to compute these values is also provided at the web link [44]. In Figure 3 we have plotted the obtained polynomials.

Remark 4.2. The process we have presented can be used for arbitrarily large $\ell$ however $\varepsilon$ should be chosen small enough (and presumably smaller and smaller as $\ell$ increase) and in that case the constants $C_{\mathcal{Q}}^{\varepsilon}$ and $C_{\mathcal{R}}^{\varepsilon}$ will degenerate. Numerical results presented Sections 5.2 and 5.3 confirm the fact that it is necessary to have $C_{\mathcal{Q}}^{\varepsilon}$ and $C_{\mathcal{R}}^{\varepsilon}$ bounded to obtain second order convergence in the norm $\|\cdot\|_{q}$. 


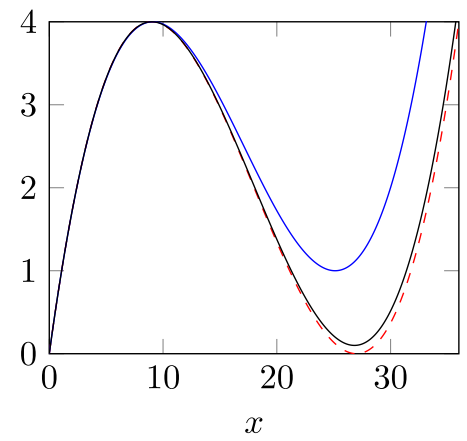

(a)

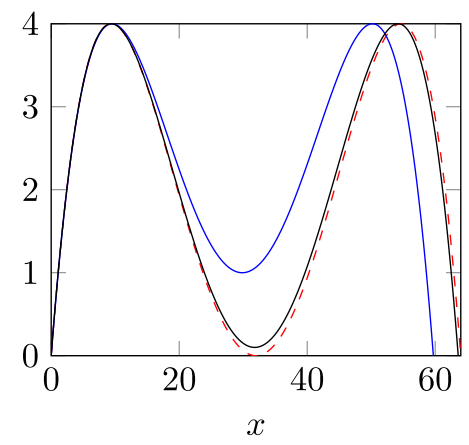

(b)

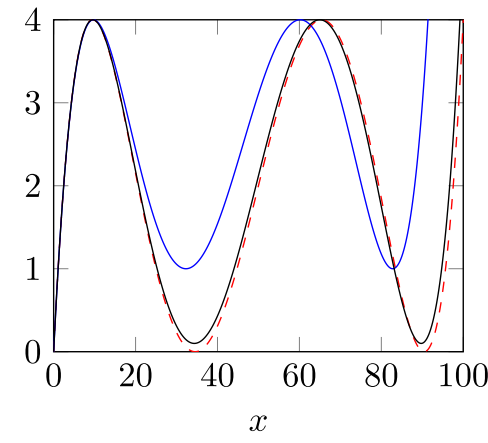

(c)

Figure 3. Plain line: Representation of $x \mathcal{P}_{p, \ell}^{\varepsilon}(x)$ for different values of $\ell$ and for $\varepsilon=0.1$ (in black) or $\varepsilon=1$ (in blue). Dashed line: Representation of $x \widetilde{\mathcal{P}}_{p, \ell}(x)$ for different values of $\ell$. (a) $l=2$. (b) $l=3$. (c) $l=4$.

\subsubsection{Algorithmic discussion}

In practice System (4.10) is solved by in three steps.

(1) Explicit computation of intermediate unknowns $u_{c, h}^{n, *}$ and $u_{f, h}^{n, *}$ by

$$
\left\{\begin{array}{l}
\frac{u_{c, h}^{n, *}-2 u_{c, h}^{n}+u_{c, h}^{n-1}}{\Delta t^{2}}+A_{c, h} u_{c, h}^{n}=f_{c, h}\left(t^{n}\right) \\
\frac{u_{f, h}^{n, *}-2 u_{f, h}^{n}+u_{f, h}^{n-1}}{\Delta t^{2}}+\mathcal{P}_{p}\left(\Delta t^{2} A_{f, h}\right)\left(A_{f, h} u_{f, h}^{n}-f_{f, h}\left(t^{n}\right)\right)=0 .
\end{array}\right.
$$

The evaluation of the term

$$
\mathcal{P}_{p, \ell}^{\varepsilon}\left(\Delta t^{2} A_{f, h}\right) A_{f, h} u_{f, h}^{n}
$$

requires $\ell+1$ evaluations of the operator $A_{f, h}$ (i.e. matrix-vector product with its algebraic representation). These evaluations can be done in two different ways, either by computing explicitly the coefficient of the polynomials $\mathcal{P}_{p, \ell}^{\varepsilon}(x)$ and using Horner algorithm, or using the second order recurrence relation of the Chebychev polynomials that can be derived from the definitions (4.14) and (4.16). For numerical stability issues, this latter choice should be privileged if $\ell$ is large, however for small values of $\ell$ both algorithms perform similarly and Horner algorithm is - in our opinion - easier to implement.

(2) Computation of $\lambda_{h}^{n}$ by solving

$$
\left(B_{c, h} B_{c, h}^{t}+B_{f, h} \mathcal{P}_{p, \ell}^{\varepsilon}\left(\Delta t^{2} A_{f, h}\right) B_{f, h}^{t}\right) \lambda_{h}^{n}=B_{c, h} u_{c, h}^{n, *}-B_{f, h} u_{f, h}^{n, *} .
$$

Note that in the context of the domain decomposition method, the Lagrange multiplier $\lambda_{h}^{n}$ corresponds to an unknown discretised on an interface and therefore the size of the system to invert in (4.21) is limited. Note also that, at the algebraic level, the corresponding matrix has a bandwidth that increases with $\ell$ and is pre-computed before iterating (see Sect. 6.2 for more details on practical algorithmic aspects).

(3) Computation of $u_{c, h}^{n+1}$ and $u_{f, h}^{n+1}$ by correction of $u_{c, h}^{n, *}$ and $u_{f, h}^{n, *}$.

$$
\left\{\begin{array}{l}
u_{c, h}^{n+1}=u_{c, h}^{n, *}-B_{c, h}^{t} \lambda_{h}^{n}, \\
u_{f, h}^{n+1}=u_{f, h}^{n, *}+\mathcal{P}_{p}\left(\Delta t^{2} A_{f, h}\right) B_{f, h}^{t} \lambda_{h}^{n} .
\end{array}\right.
$$


This step involves $\ell$ evaluations of the operator $A_{f, h}$. This is clearly an over-cost compared to an ideal local time stepping strategy. Note however that the term $B_{f, h}^{t} \lambda_{h}^{n}$ may have a small support, this property is used in practice to reduce the amount of computations needed in this step.

To summarize, the main complexity of the algorithm comes from - at each time iteration - the product with the operator $A_{c, h}, 2 \ell+1$ products with $A_{f, h}$ and the solving step of (4.21). The time step being relaxed $\beta_{\ell}^{\varepsilon} \simeq \ell+1$ times, the proposed algorithm is especially efficient when the operator $A_{c, h}$ is more costly to evaluate than the operator $A_{f, h}$.

The proposed algorithmic complexity is the same as in the local time stepping introduced in [17]. The strategy presented in [19] required a smaller number of matrix-vector product is required and no system must be solved. Therefore, in the same configuration (same mesh and same time step for which both methods are stable), the algorithm proposed in [19] should be more efficient. However, this method does not rely on a decomposition domain approach and has yet to be extended to transmission problems between non-conformal meshes. For these reasons, definitive comparisons between both methods are difficult.

Remark 4.3. Note that although the size of the matrix system corresponding to equation (4.21) may be relatively small (it is reduced to the Lagrange multiplier unknown on the interface) the matrix may become full for large $\ell$. In that case the local implicit scheme of Section 4.1 may be preferred.

\section{Numerical illustrations in 1D}

The computational code used to obtain the results of this section is available as supplementary material at the web link [44].

In this section we present numerical results in 1D that illustrate the convergence behavior of the schemes we have proposed. We consider the wave equation (2.1), with homogeneous Neumann boundary condition, posed on the domain $\Omega=(-0.5,0.5)$ with $\Omega_{c}=(-0.5,0), \Omega_{f}=(0,0.5)$ and $\Sigma=\{0\}$. We assume that $\mu_{c}=1$ and we denote $\mu \equiv \mu_{f} \leq 1$. Note that $\mu$ is therefore a measure of the contrast between the two subdomains.

We consider the propagation problem of a pulse generated by a source term. More precisely, initial data are null and the source term is computed so that the exact solution is given by, for $t \in[0, T]$ with $T=0.5$,

$$
u(x, t)=\left\{\begin{array}{ll}
u_{c}(x, t) & x<0 \\
u_{f}(x, t) & x>0
\end{array}= \begin{cases}h\left(\frac{t-\tau}{t_{0}}\right) r(x-t)+\mathcal{R} r(-x-t) & x<0 \\
\mathcal{T} r(x-\sqrt{\mu} t) & x>0\end{cases}\right.
$$

with $r(x)=1_{\left[x_{0}-\sigma, x_{0}+\sigma\right]} e^{-2 /\left(1-\left(x-x_{0}\right)^{2} / \sigma^{2}\right)}$ (a smooth compactly supported pulse), $x_{0}=-0.25, \sigma=0.05$ and

$$
\begin{aligned}
& \mathcal{R}=\frac{1-\sqrt{\mu}}{1+\sqrt{\mu}} \quad \text { and } \mathcal{T}=1+\mathcal{R}=2 \frac{1}{1+\sqrt{\mu}}, \\
& \tau=0.025, \quad t_{0}=0.0625, \quad h(t)= \begin{cases}0 & t \leq 0, \\
1 & t \geq 1, \\
\frac{1}{1+e^{\frac{1}{t}+\frac{1}{t-1}}} & 0<t<1 .\end{cases}
\end{aligned}
$$

Qualitatively speaking the sought solution corresponds after time $t>\tau+t_{0}$ to a right propagating pulse that is solution to a transport problem - with no source term - before it reaches the interface $\Sigma$.

For the space discretisation we use standard second order Galerkin finite elements with a lumped mass matrix (see for instance [10-12]) on a uniform mesh of $\Omega_{c}$ and $\Omega_{f}$ and we denote $h_{c}$ and $h_{f}$ the respective mesh sizes and $q_{f}$ the refinement rate. We have s

$$
h_{c}=h, \quad h_{f}=\frac{h}{q_{f}} \quad \text { and } \quad \frac{\rho_{f, h}}{\rho_{c, h}}=\mu q_{f}^{2} .
$$




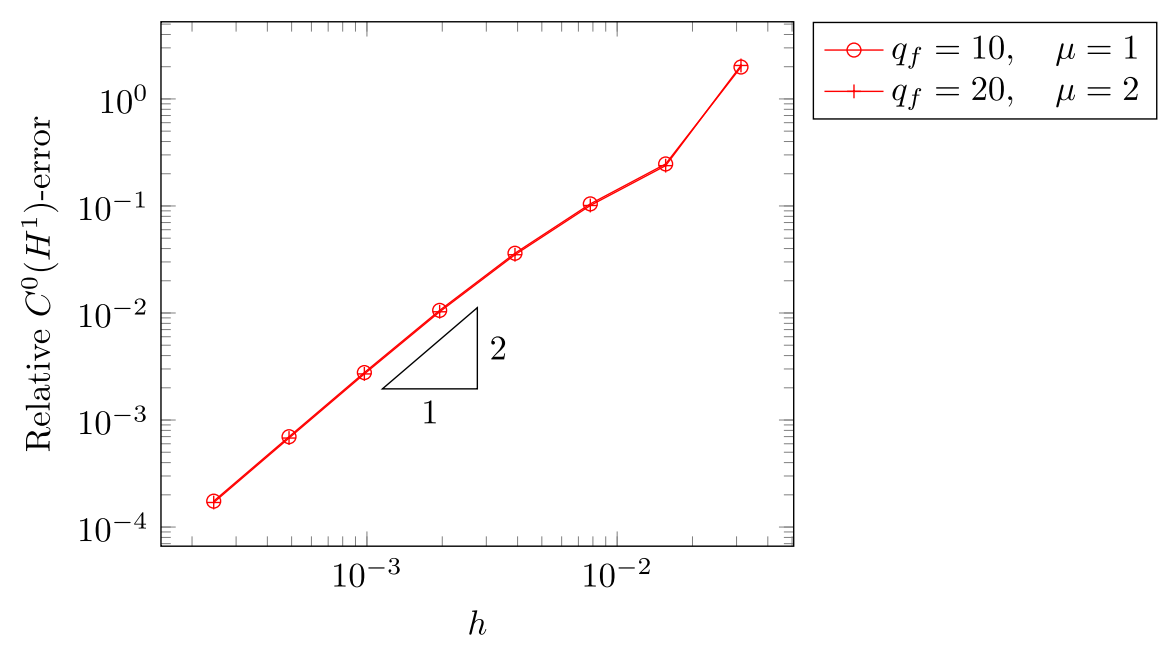

FiguRE 4. Space-time convergence plot for local implicit schemes for different values of $\left(\mu, q_{f}\right)$.

The chosen space discretisation provides a second order convergence in space for the $H^{1}$-norm (see [23]).

We recall that $\Delta t=2 \alpha / \sqrt{\rho_{c, h}}$ for some $0<\alpha \leq 1$. In what follows, we plot space-time convergence curves by setting $\alpha$ to some given values, and computing the solution to the discrete problem for some sequence $h$ going to zero (this implies that $\Delta t$ goes to zero accordingly). Then the discrete solution $\left(u_{c, h}^{n}, u_{f, h}^{n}\right)$ is compared to the analytic expression (5.1) and we plot

$$
\frac{\sup _{n \Delta t \leq T}\left\|\mathcal{I}_{c, h} u_{c}\left(t^{n}\right)-u_{c, h}^{n}\right\|_{c}}{\sup _{n \Delta t \leq T}\left\|\mathcal{I}_{c, h} u_{c}\left(t^{n}\right)\right\|_{c}}+\frac{\sup _{n \Delta t \leq T}\left\|\mathcal{I}_{f, h} u_{f}\left(t^{n}\right)-u_{f, h}^{n}\right\|_{f}}{\sup _{n \Delta t \leq T}\left\|\mathcal{I}_{f, h} u_{f}\left(t^{n}\right)\right\|_{f}}
$$

where $\mathcal{I}_{c, h}$ and $\mathcal{I}_{f, h}$ denote the interpolation operators on the nodal finite element spaces.

\subsection{Local implicit scheme}

In order to assess numerically the behavior of local implicit schemes described in Section 4.1, we set $\mathcal{P}_{k}(x)=1$ and $\mathcal{P}_{p}(x)=1$. More specifically, this means that the left-hand side of the domain is discretized with an explicit leap-frog scheme, while the right-hand side of the domain is discretized with an unconditionally stable implicit $\theta$-scheme with $\theta=1 / 4$. We have chosen the optimal value $\alpha=1$ and the values for the refinement ratio and the ratio of velocity $\left(q_{f}, \mu\right)=(10,1)$ and $\left(q_{f}, \mu\right)=(20,2)$.

This configuration is difficult since the constraint on the time step is severe (a pure explicit case would require a time step around 10 to 30 smaller). Nevertheless, the convergence plots represented in Figure 4 show that a second order rate of convergence is achieved. In fact this was to be expected since Corollary 3.8 can be applied with $C_{\mathcal{Q}}=0$ and $C_{\mathcal{R}}=1$.

\subsection{Stabilised explicit scheme}

In order to assess the behavior of the stabilised explicit scheme described in Section 4.2, we set

$$
\mathcal{P}_{p}(x)=1-\frac{x}{16} \quad \text { and } \quad \mathcal{P}_{k}(x)=1-x \frac{\mathcal{P}_{p}(x)}{4}=1-\frac{x}{4}+\frac{x^{2}}{64} .
$$

We first investigate the situation of a homogeneous medium $(\mu=1)$ where the subdomain $\Omega_{f}$ is refined by a factor $q_{f}=2$. We make the value of $\alpha$ increase from $\alpha=0.9$ to 1 . As stated in Section 4.2 , the value $\alpha=1$ prevents us from applying Corollary 3.9 since the values of $C_{\mathcal{R}}$ and $C_{\mathcal{Q}}$ blow up when $\alpha$ approaches 1 . The 


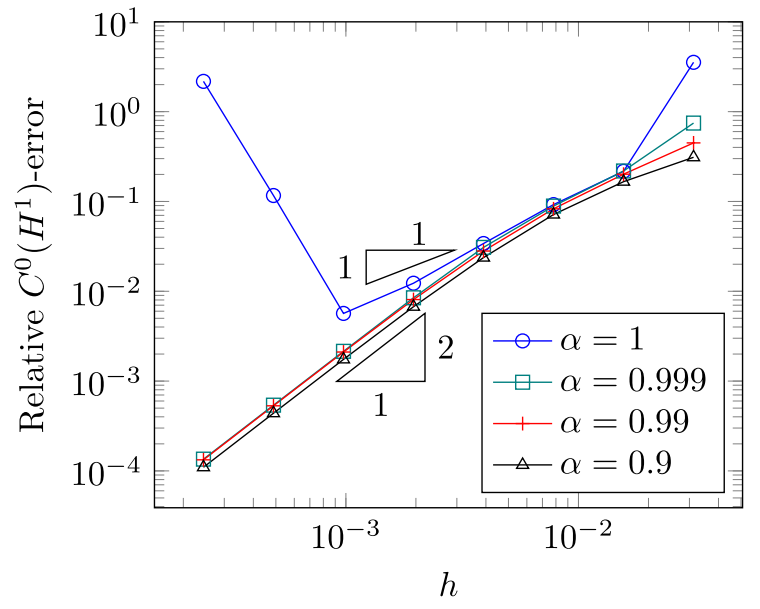

(a)

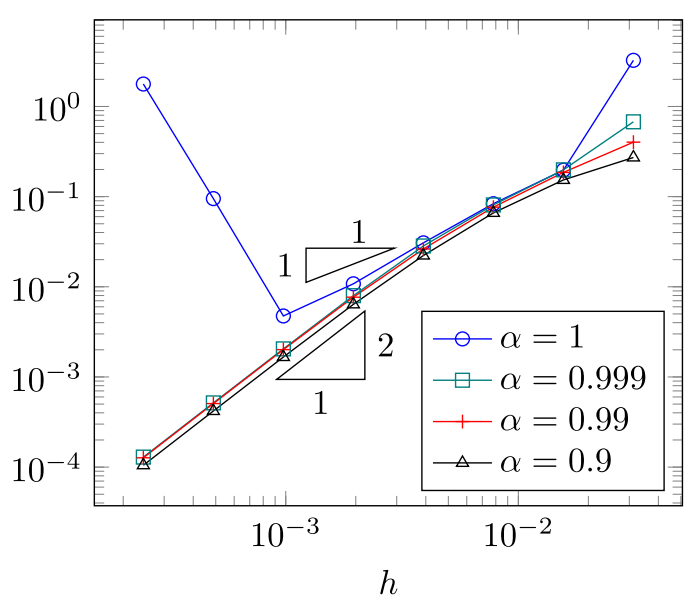

(b)

FIGURE 5. Space-time convergence plots for the stabilised explicit scheme for different values of $\left(\alpha, \mu, q_{f}\right)$. (a) $\mu=1$ and $q_{f}=2$. (b) $\mu=0.25$ and $q_{f}=4$.

numerical results displayed in Figure 5a show that values of $\alpha$ very close to 1 (up to 0.999) give the expected convergence rate of 2 , and that indeed, choosing $\alpha=1$ does not lead to a second order space/time convergence (the convergence is of order 1 before diverging). As a second example, we consider an inhomogeneous medium with $\mu=0.25$, we choose $q_{f}=4$, and we perform the same numerical tests. As observed in Figure $5 \mathrm{~b}$, the same conclusions can be drawn.

\subsection{Local time stepping using the Leap-Frog Chebychev method}

In order to assess the behavior of the schemes constructed in Section 4.3, we set, for a given $\varepsilon, \mathcal{P}_{p}(x)=\mathcal{P}_{p, \ell}^{\varepsilon}(x)$ according to the values given in Table 1 or $\mathcal{P}_{p}(x)=\widetilde{\mathcal{P}}_{\ell}(x)$ and we always choose $\mathcal{P}_{k}(x)$ as in (4.9) meaning that we are using explicit schemes. We consider $\mu=1$, and $q_{f}=3$ or $q_{f}=4$.

According to equation (5.2) we have $\rho_{f, h}=q_{f}^{2} \rho_{c, h}$. The polynomial $\mathcal{P}_{p, \ell}^{\varepsilon}$ is chosen such that $\ell=q_{f}-1$ and $\varepsilon=0.1$, and the value of $\alpha \equiv \alpha^{\varepsilon}$ is chosen as

$$
\alpha^{\varepsilon}=\beta_{\ell}^{\varepsilon} \sqrt{\frac{\rho_{c, h}}{\rho_{f, h}}}=\frac{\beta_{\ell}^{\varepsilon}}{q_{f}}
$$

where the values of $\beta_{\ell}^{\varepsilon}$ are given in Table 1 . This choices of parameters ensure that the stability condition is fulfilled. In Figure 6, we have displayed the convergence obtained. In all the cases we observe second order space-time convergence with the polynomials $\mathcal{P}_{p}(x)=\mathcal{P}_{p, \ell}^{\varepsilon}(x)$ while the choice $\mathcal{P}_{p}(x)=\widetilde{\mathcal{P}}_{\ell}(x)$ only gives a first order convergence behavior.

\section{Numerical illustrations in 2D}

In this section we present numerical results in $2 \mathrm{D}$ that illustrate the convergence behavior of the scheme (4.10) as well as the performance gain one could achieved with such method with $\mathcal{P}_{p}=\mathcal{P}_{\ell}^{\varepsilon}$ where $\ell \in\{2,3\}$ and $\varepsilon=0.1$. We systematically use Galerkin high order spectral finite elements on quadrangles (see [10]) for the construction of the spaces $V_{c, h}$ and $V_{f, h}$, whereas for the construction of $L_{h}$ we use discontinuous nodal finite elements (on Gauss points, see [29]) on the edges defined by the intersection of the boundary $\Sigma$ and the coarse mesh of $\Omega_{c}$. Numerical results are obtained with an in-house solver developed in $\mathrm{C}++$, parallelization is done using OpenMP and Eigen [43] is used as a linear algebra library. 


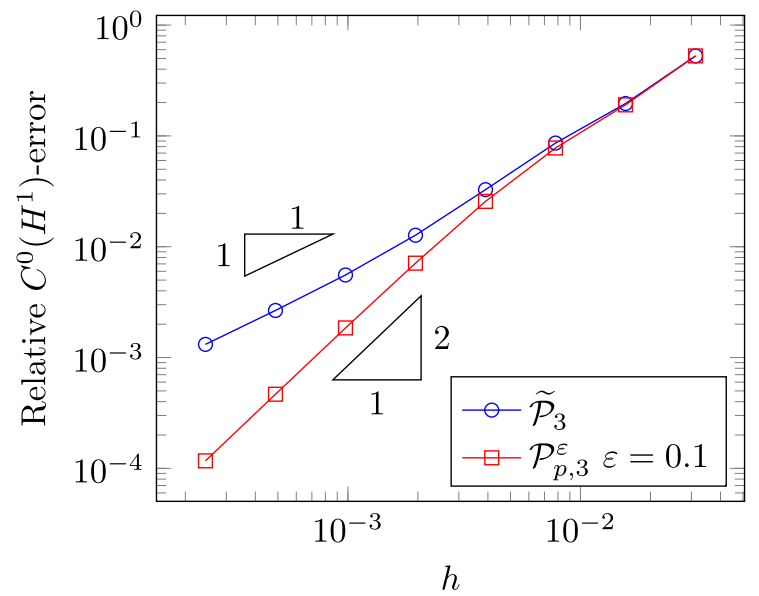

(a)

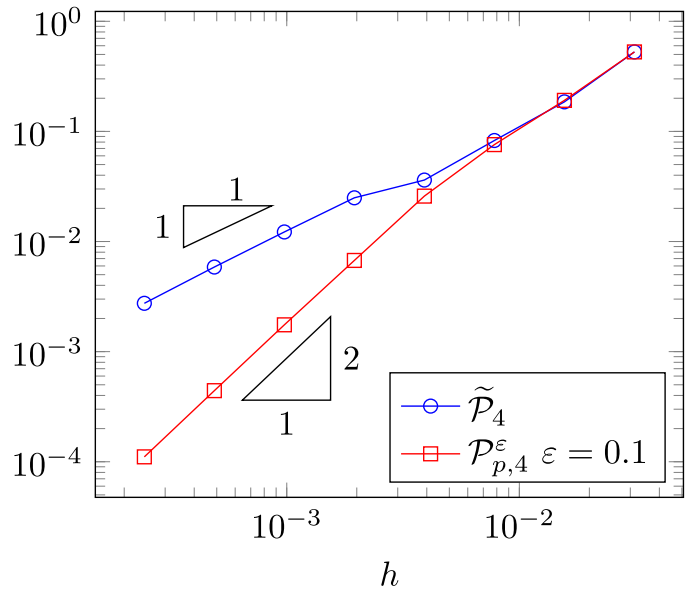

(b)

FIgURE 6. Space-time convergence plots for the local time stepping explicit scheme for different values of $q_{f}$ and $\mathcal{P}_{p}(x)$. (a) $q_{f}=3, \alpha=0.9960$. (b) $q_{f}=4, \alpha=0.9960$.

\subsection{Convergence result}

We consider the wave equation (2.1), with homogeneous Neumann boundary condition, posed on the domain $\Omega=(0,1)^{2}$ and $\Omega_{f}=(0.6,0.7)^{2}$. We assume that $\mu_{c}=1$ and $\mu_{f}=1$, so that the domain decomposition is artificial, but allow us to compare the simulations to an accurate reference solution. We consider the propagation of initial data. More precisely, the source term is null and the initial data are computed so that the reference solution is given by, for $t \in[0, T]$ with $T=0.35$,

$$
u(x, y, t)=g(x, y, t+0.1)
$$

where $g$ is the solution to the wave propagation problem

$$
\partial_{t}^{2} g-\Delta g=0, \quad g(\cdot, 0)=0 \text { and } \partial_{t} g(\cdot, 0)=\exp \left(-\alpha(x-0.5)^{2}-\alpha(y-0.5)^{2}\right) \text { in } \mathbb{R}^{2} \text { with } \alpha=1000 .
$$

The solution $g$ can be computed semi-analytically using the expression of the Green's function for the wave equation in $\mathbb{R}^{2}$.

The meshes of $\Omega_{c}$ and $\Omega_{f}$ are discretised uniformly with $h_{f} / h_{c}=8$. The order used in $\Omega_{c}$ (resp. $\Omega_{f}$ ) is 8 (resp. 4). Consequently, the meshes are not conform. Order 7 is used to construct the space $L_{h}$. Using the macro-element analysis of [28] one can check that (2.10) holds. Indeed it is sufficient to check an injectivity property on a reference configuration (see Eq. (30) in [28]). In our case, such injectivity property is guaranteed by the high number of test functions of $V_{h, f}$ on the transmission boundary. The time step is chosen as

$$
\Delta t^{2}=\min \left(\frac{4 \alpha}{\tilde{\rho}_{c, h}}, \frac{4 \alpha \beta_{\ell}^{2}}{\tilde{\rho}_{f, h}}\right)
$$

where $\alpha=0.99$ and $\left(\tilde{\rho}_{c, h}, \tilde{\rho}_{f, h}\right)$ are approximations of $\left(\rho_{c, h}, \rho_{f, h}\right)$ computed by power iteration. The approximated spectral radii are close enough to the exact spectral radii such that (6.2) implies the stability condition (4.13). Figure 7 displays a snapshot of the solution at different times. The relative error is computed at the final simulated time. Table 2 shows the relative errors as the discretisation diminishes. A second order convergence rate is observed. 

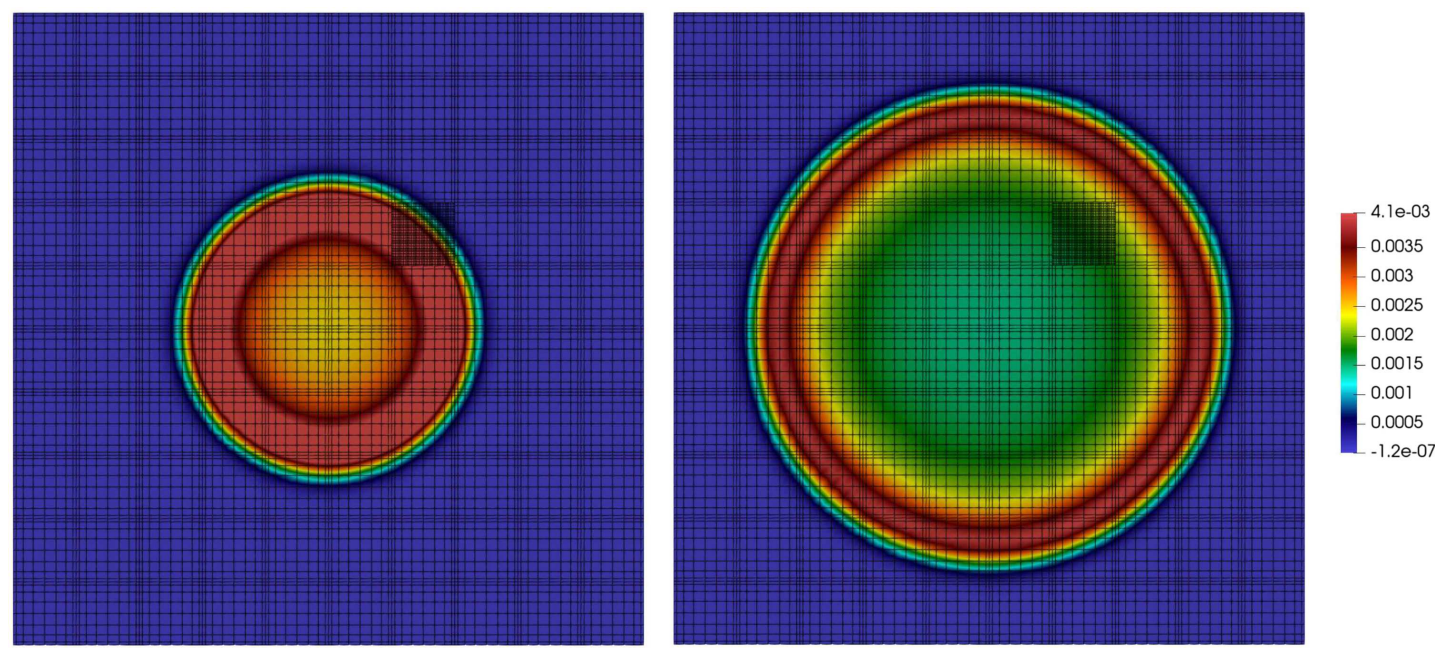

Figure 7. Snapshot of the solution at times 0.2 and 0.35 (up to a $\Delta t$ ). Order 8 elements are used on the coarse domain whereas order 4 elements are used on the fine one (for visualisation, high-order solutions are interpolated on a low order finite element space using a splitted mesh).

TABLE 2. Convergence result: parameters of the numerical experiments.

\begin{tabular}{lllll}
\hline \hline$h_{c}$ & 0.1 & 0.05 & 0.025 & 0.0125 \\
$h_{f}$ & 0.0125 & 0.00625 & 0.003125 & 0.0015625 \\
$\sqrt{\rho_{f, h} / \rho_{c, h}}$ & 2.3 & 2.3 & 2.3 & 2.3 \\
$\Delta t$ & 0.0030 & 0.0015 & 0.00075 & 0.00038 \\
$\frac{\left\|I_{c, h} u_{c}\left(t^{N}\right)-u_{c, h}^{N}\right\|_{c}}{\left\|I_{c, h} u_{c}\left(t^{N}\right)\right\|_{c}}$ & 0.029 & 0.0074 & 0.0019 & 0.00053 \\
Conv. order & - & 1.98 & 1.97 & 1.79 \\
\hline
\end{tabular}

\subsection{Performance illustration}

The chosen configuration is the same as in the previous section expect that the domain $\Omega$ differs. Indeed, in this configuration the domain $\Omega_{f}$ includes a circular hole that is meshed with quadrangles (see Figs 8 and 9). Order 10 finite elements are used in $\Omega_{c}$ while order 9 are used to construct the space $L_{h}$ and second order finite elements are used in $\Omega_{f}$. We aim at comparing the computational cost of two schemes: (4.10) with $\mathcal{P}_{p}=\mathcal{P}_{2}^{\varepsilon}$ and with $\mathcal{P}_{p}=\mathcal{P}_{3}^{\varepsilon}$ where $\varepsilon=0.1$ (the time step being chosen by formula (6.2)) and (4.10) with $\mathcal{P}_{p}$ equals to the polynomial 1 . In this latter case the scheme reads

$$
\left\{\begin{array}{l}
\frac{u_{c, h}^{n+1}-2 u_{c, h}^{n}+u_{c, h}^{n-1}}{\Delta t^{2}}+A_{c, h} u_{c, h}^{n}+B_{c, h}^{t} \lambda_{h}^{n}=0 \\
\frac{u_{f, h}^{n+1}-2 u_{f, h}^{n}+u_{f, h}^{n-1}}{\Delta t^{2}}+A_{f, h} u_{f, h}^{n}-B_{f, h}^{t} \lambda_{h}^{n}=0 \\
B_{c, h} u_{c, h}^{n}=B_{f, h} u_{f, h}^{n}
\end{array}\right.
$$




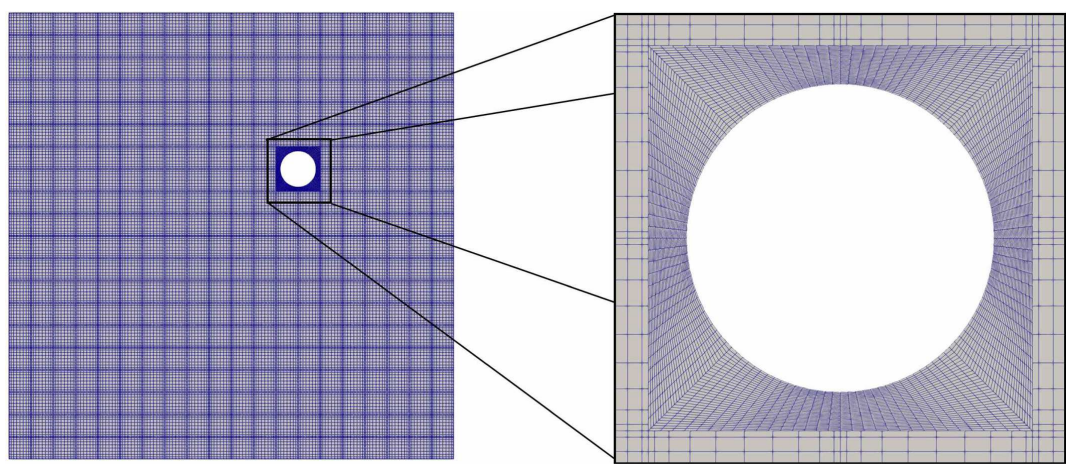

FiguRE 8. Meshes used for the configuration with a circular defect.
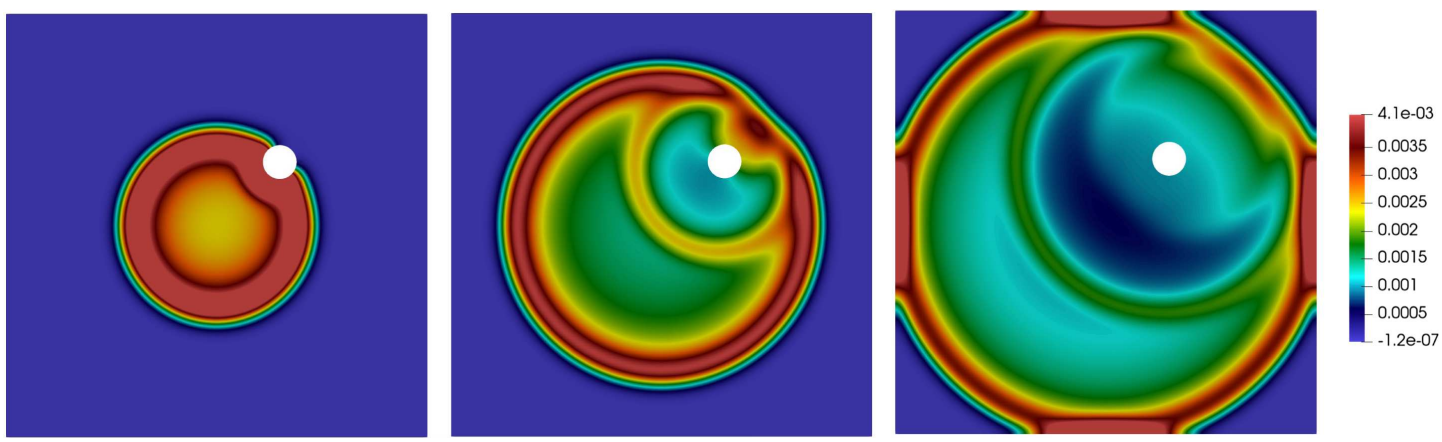

Figure 9. Snapshot of the solution at times $0.2,0.35$ and 0.50 (up to a $\Delta t$ ).

and corresponds to the straightforward discretization of (2.8) by a Leap-frog approach. For (6.3) the time step is chosen as

$$
\Delta t^{2}=\min \left(\frac{4 \alpha}{\tilde{\rho}_{c, h}}, \frac{4 \alpha}{\tilde{\rho}_{f, h}}\right),
$$

with $\alpha=0.99$. To compute the new iterate with either choice of polynomial we follow the approach described in Section 4.3.4.

The simulation is run on a long time period $(T=10)$ and time spent in each steps (see Tab. 3) of the algorithm are measured. There are five measured steps:

(0) Computation of the matrix involved in (4.21) as well as the time spend to compute its factorization.

(1c) Prediction in $\Omega_{c}$. Computation of $u_{c, h}^{n, *}$ in Step 1 of the algorithm described Section 4.3.4.

(1f) Prediction in $\Omega_{f}$. Computation of $u_{f, h}^{n, *}$ in the same step.

(2) Transmission. Computation of $\lambda_{h}^{n}$ by solving System (4.21).

(3c) Correction in $\Omega_{c}$. Computation of $u_{c, h}^{n+1}$ thanks to the first equation of (4.22).

(3f) Correction in $\Omega_{f}$. Computation of $u_{f, h}^{n+1}$ thanks to the second equation.

We give in Table 3 the parameters of the experiments as well as the measured times spent in each steps (in relative values). In the specific chosen configuration the proposed algorithms reaches the final simulation time, approximately 2.27 (resp. 2.63) times faster for $\ell=2$ (resp. $\ell=3$ ). One can see in Table 3 that the time spent in the computation of $u_{f, h}^{n, *}$ is constant for the three methods whereas the time spent to compute $u_{c, h}^{n, *}, u_{c, h}^{n+1}$ and $\lambda_{h}^{n}$ decreases by a factor 3 and 4 approximately which is a consequence of the time step increase. Finally 
TABLE 3. Performance illustration: parameters of the numerical experiments and relative time spent by the algorithm in each steps.

\begin{tabular}{ll}
\hline \hline $\operatorname{Dim}\left(V_{c, h}\right)$ & 40040 \\
$\operatorname{Dim}\left(V_{f, h}\right)$ & 4224 \\
$\operatorname{Dim}\left(L_{h}\right)$ & 80 \\
$\tilde{\rho}_{c, h}$ & $3.98 \times 10^{6}$ \\
$\tilde{\rho}_{f, h}$ & $9.69 \times 10^{7}$ \\
\hline
\end{tabular}

\begin{tabular}{llll}
\hline \hline Scheme (4.10) & $\mathcal{P}_{p}=1$ & $\mathcal{P}_{p}=\mathcal{P}_{2}^{0.1}$ & $\mathcal{P}_{p}=\mathcal{P}_{3}^{0.1}$ \\
\hline$\Delta t$ & $2.01 \times 10^{-4}$ & $6.01 \times 10^{-4}$ & $8.01 \times 10^{-4}$ \\
$N$ & 49711 & 16637 & 12478 \\
Step 0 & $4.3 \times 10^{-5}$ & $2.5 \times 10^{-4}$ & $3.4 \times 10^{-4}$ \\
Step 1c & 0.61 & 0.20 & 0.16 \\
Step 1f & 0.11 & 0.10 & 0.10 \\
Step 2 & 0.041 & 0.013 & 0.010 \\
Step 3c & 0.22 & 0.072 & 0.055 \\
Step 3f & 0.024 & 0.047 & 0.053 \\
Total & 1 & 0.44 & 0.38 \\
\hline
\end{tabular}

note that the time spent in Step 3f (computation of $u_{f, h}^{n+1}$ ) increases with $\ell$ without penalizing too much the performances, so does Step 0 (computation and factorization of the matrix used in Step 2). This latter step representing a marginal effort in term of computations.

To sum-up, this case well illustrates the actual performance gain one can expect with this method. Note that this gain tends to 3 (resp. 4 ) for $\ell=2$ (resp. 3) as $\operatorname{Dim}\left(V_{f, h}\right) / \operatorname{Dim}\left(V_{c, h}\right)$ tends to zero.

\section{COMPARISONS With EXISTING APPROaches}

\subsection{The fully explicit Local Time Stepping of [26]}

The computational code used to obtain the results of this section is available as supplementary material at the web link [44].

In $[19,26]$, an explicit Local Time Stepping Algorithm is proposed and is proved to be second order convergent for the $L^{2}$-norm. It is used in the context of solving the following semi-discrete wave equation:

$$
\frac{\mathrm{d}^{2}}{\mathrm{~d} t^{2}} u_{h}+A_{h} u_{h}=f_{h}
$$

From formula (12) of [26] with $p=2$ one can derive the following scheme

$$
\frac{u_{h}^{n+1}-2 u_{h}^{n}+u_{h}^{n-1}}{\Delta t^{2}}+\left(A_{h}-\frac{\Delta t^{2}}{16} A_{h} P_{h} A_{h}\right) u_{h}^{n}=f_{h}^{n},
$$

where $P_{h}: V_{h} \mapsto V_{h}$ is a restriction operator on a region discretised with a fine grid (with an overlap of one element in our computations). Notice that this algorithm amounts to solving a leap-frog scheme for the kernel of $P_{h}$ and to a modified scheme for the complement. Moreover, from Algorithm 1 (page 1000) of [26] we can deduce a variant of $(\mathrm{EX}-2 \mathrm{~b})$,

$$
\frac{u_{h}^{n+1}-2 u_{h}^{n}+u_{h}^{n-1}}{\Delta t^{2}}+\left(A_{h}-\frac{\Delta t^{2}}{16} A_{h} P_{h} A_{h}\right) u_{h}^{n}=\tilde{f}_{h}^{n}-\frac{\Delta t^{2}}{16} A_{h} P_{h} f_{h}^{n}
$$

where $\tilde{f}_{h}^{n}$ can be defined in two ways, denoting $f_{h}^{n+\frac{1}{2}}=f_{h}\left(t^{n}+\Delta t / 2\right)$ we set

$$
\tilde{f}_{h}^{n}=\frac{1}{4} P_{h}\left(f_{h}^{n+\frac{1}{2}}+2 f_{h}^{n}+f_{h}^{n-\frac{1}{2}}\right)+\left(I_{h}-P_{h}\right) f_{h}^{n} \quad(a) \quad \text { or } \quad \tilde{f}_{h}^{n}=f_{h}^{n} \quad(b) .
$$



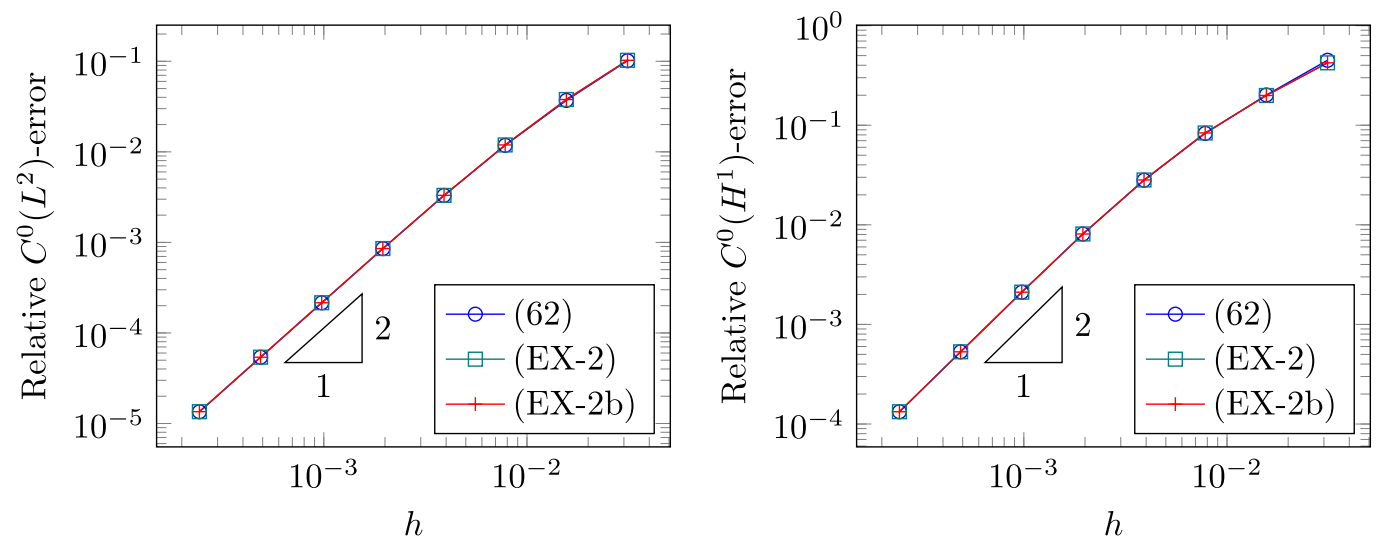

FIGURE 10. Space-time convergence plots for the LTS explicit scheme. The analytical solution is a propagating pulse.

The choice (7.2a) gives exactly the Algorithm 1 of [26] while the more simple choice (7.2b) gives similar observed convergence behavior and so will be used in what follows.

In the following we present a numerical assessment, in a one-dimensional setting, of local time stepping procedures that have the same computational cost: the stabilised explicit scheme (4.6) of Section 4.2, the scheme (EX-2) and its variant (EX-2b).

The considered case is the same as in Section 5 with $\mu=1$ (homogeneous medium). More precisely, we solve up to time $T=0.5$, the equation

$$
\partial_{t}^{2} u-\partial_{x}^{2} u=f, \quad x \text { in }(-0.5,-0.5),
$$

with homogeneous Neumann boundary condition. The discretisation parameters are $\alpha=0.99$ and $q_{f}=2$. The purpose of these tests is to quantify the relative $L^{2}$ and $H^{1}$-errors with respect to three chosen continuous analytical solutions associated with adequate source terms or initial data.

\subsubsection{Propagating pulse}

The first considered case is a propagating pulse as described in Section 5. In Figure 10 are displayed the relative $L^{2}$ and $H^{1}$-errors between the solutions of the three numerical schemes (Scheme (4.6), (EX-2) and $(\mathrm{EX}-2 \mathrm{~b})$ ) and the analytical solution, with respect to the mesh size $h$ (note that $\Delta t$ goes to zero with $h$ because of Assumption 3.2).

\subsubsection{Quasi-static solution}

We choose vanishing initial data for the wave equation (7.3) and choose $f$ such that the solution is smooth and given by

with $\tau=0.1, t_{0}=0.25$ and

$$
u(x, t)=g(x) h\left(\frac{t-\tau}{t_{0}}\right)
$$

$$
g(x)= \begin{cases}x^{2}\left(x+\frac{1}{2}\right)^{2} & x<0 \\ x^{2}\left(x-\frac{1}{2}\right)^{2} & x>0\end{cases}
$$

Note that the solution reaches a static state after time $t \geq 0.35$. The obtained convergence curves are displayed in Figure 11. One can see that the three schemes behave similarly in terms of convergence in the $L^{2}$-norm, however the scheme (EX-2b) is less accurate in the $H^{1}$-norm. More precisely, half an order of convergence is lost. 

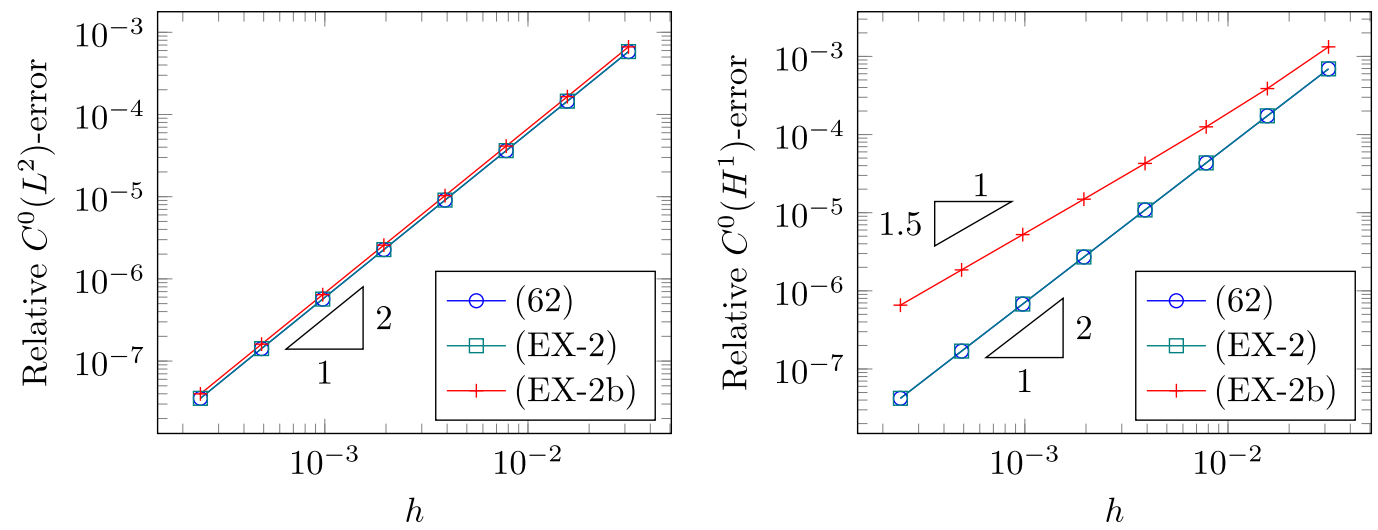

FIGURE 11. Space-time convergence plots for the LTS explicit schemes. The analytical solution is static for $t \geq 0.35$.
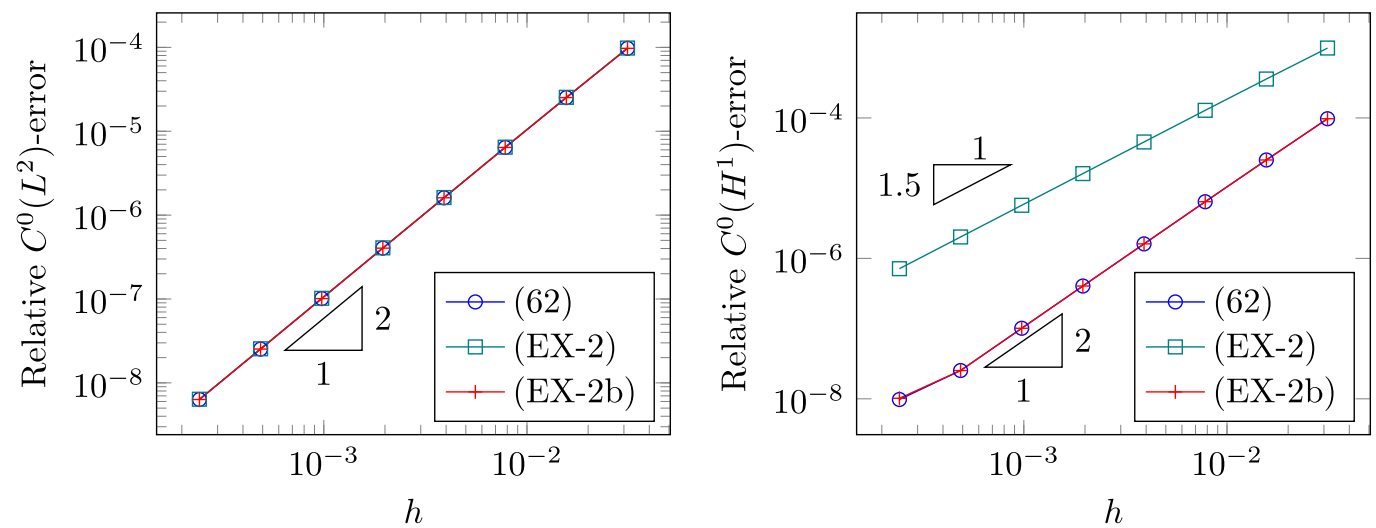

FIGURE 12. Space-time convergence plots for the LTS explicit schemes. The analytical solution is constant in space.

\subsubsection{Spatially constant solution}

We choose vanishing initial data for the wave equation (7.3) and choose $f$ such that the solution is smooth and given by

$$
u(x, t)=h\left(\frac{t-\tau}{t_{1}}\right) .
$$

with $\tau=0.1$ and $t_{1}=0.8$. The analytical solution is therefore constant in space. The obtained convergence curves are displayed in Figure 12. Again, one the one hand, one can see that the three schemes behave similarly in terms of convergence in the $L^{2}$-norm. On the other hand, it is this time the scheme (EX-2) which is less accurate in the $H^{1}$-norm (half an order of convergence is lost).

\subsection{The implicit LTS of [17]}

In equations (13.74)-(13.76) of [17], we find an algebraic formulation for conservative local time stepping. This formulation is written for the system of elastodynamics written in a first order form in time. However, by elimination of the variable corresponding to the velocity, one can show that the algebraic formulation is 
equivalent to the following system

$$
\left\{\begin{array}{l}
M_{\sigma, c} \frac{\Sigma_{c}^{2 n+1}-2 \Sigma_{c}^{2 n-1}+\Sigma_{c}^{2 n-3}}{(2 \Delta t)^{2}}+K_{c} \Sigma_{c}^{2 n-1}-C_{c}^{*} \frac{J^{2 n+1}-J^{2 n-3}}{4 \Delta t}=0 \\
M_{\sigma, f} \frac{\Sigma_{f}^{2 n+1}-2 \Sigma_{f}^{2 n}+\Sigma_{f}^{2 n-1}}{\Delta t^{2}}+K_{f} \Sigma_{f}^{2 n}-C_{f}^{*} \frac{J^{2 n+1}-J^{2 n-1}}{2 \Delta t}=0 \\
M_{\sigma, f} \frac{\Sigma_{f}^{2 n}-2 \Sigma_{f}^{2 n-1}+\Sigma_{f}^{2 n-2}}{\Delta t^{2}}+K_{f} \Sigma_{f}^{2 n-1}-C_{f}^{*} \frac{J^{2 n+1}-J^{2 n-3}}{4 \Delta t}=0 . \\
C_{c} \Sigma_{c}^{2 n+1}+C_{f} \Sigma_{f}^{2 n+1}=0 . \\
u_{c}
\end{array}\right.
$$

In this formulation the unknowns $\Sigma_{c}^{n}$ and $\Sigma_{f}^{n}$ are vectors corresponding to stresses in a coarse and fine region respectively and, $J^{n}$ are vectors corresponding to normal stresses, $K_{c}$ and $K_{f}$ are stiffness matrices (equal respectively to $B_{c}^{*} M_{v, c}^{-1} B_{c}$ and $B_{f}^{*} M_{v, c}^{-1} B_{f}$ with the notation given in [17]), $M_{\sigma, c}$ and $M_{\sigma, f}$ are mass matrices. Now we aim at eliminating intermediate steps in the evaluation for the fine region, more precisely, the sequence of even iterates $\left\{\Sigma_{f}^{2 n}\right\}$ for $n \geq 0$. To do so, we write (7.4b) centered at time $t^{2 n}$ and subtract two times equation (7.4c) centered around $t^{2 n-1}$ and add the equation (7.4b) centered at time $t^{2 n-2}$. We obtain

$$
M_{\sigma, f} \frac{\Sigma_{f}^{2 n+1}-4 \Sigma_{f}^{2 n}+6 \Sigma_{f}^{2 n-1}-4 \Sigma_{f}^{2 n-2}+\Sigma_{f}^{2 n-3}}{\Delta t^{2}}+K_{f}\left[\Sigma_{f}^{2 n}-2 \Sigma_{f}^{2 n-1}+\Sigma_{f}^{2 n-2}\right]=0 .
$$

Now we use the fact that

$$
\frac{\Sigma_{f}^{2 n+1}-4 \Sigma_{f}^{2 n}+6 \Sigma_{f}^{2 n-1}-4 \Sigma_{f}^{2 n-2}+\Sigma_{f}^{2 n-3}}{\Delta t^{2}}=\frac{\Sigma_{f}^{2 n+1}-2 \Sigma_{f}^{2 n-1}+\Sigma_{f}^{2 n-3}}{\Delta t^{2}}-\frac{4}{\Delta t^{2}}\left[\Sigma_{f}^{2 n}-2 \Sigma_{f}^{2 n-1}+\Sigma_{f}^{2 n-2}\right],
$$

and therefore we obtain

$$
M_{\sigma, f} \frac{\Sigma_{f}^{2 n+1}-2 \Sigma_{f}^{2 n-1}+\Sigma_{f}^{2 n-3}}{\Delta t^{2}}+\left[K_{f}-\frac{4}{\Delta t^{2}} M_{\sigma, f}\right]\left(\Sigma_{f}^{2 n}-2 \Sigma_{f}^{2 n-1}+\Sigma_{f}^{2 n-2}\right)=0 .
$$

Now using (7.4c), we replace the quantity inside the parenthesis

$$
M_{\sigma, f} \frac{\Sigma_{f}^{2 n+1}-2 \Sigma_{f}^{2 n-1}+\Sigma_{f}^{2 n-3}}{\Delta t^{2}}+\Delta t^{2}\left[K_{f}-\frac{4}{\Delta t^{2}} M_{\sigma, f}\right]\left(-M_{\sigma, f}^{-1} K_{f} \Sigma_{f}^{2 n-1}+M_{\sigma, f}^{-1} C_{f}^{*} \frac{J^{2 n+1}-J^{2 n-3}}{4 \Delta t}\right)=0 .
$$

Dividing by 4 the previous equation and rearranging terms we obtain

$$
M_{\sigma, f} \frac{\Sigma_{f}^{2 n+1}-2 \Sigma_{f}^{2 n-1}+\Sigma_{f}^{2 n-3}}{(2 \Delta t)^{2}}+\left[I_{f}-\frac{(2 \Delta t)^{2}}{16} K_{f} M_{\sigma, f}^{-1}\right]\left[K_{f} \Sigma_{f}^{2 n-1}-C_{f}^{*} \frac{J^{2 n+1}-J^{2 n-3}}{4 \Delta t}\right]=0
$$

where $I_{f}$ is the identity matrix in the appropriate vector space. Let us denote

$$
U_{f}^{n}:=\Sigma_{f}^{2 n-1}, \quad U_{c}^{n}:=\Sigma_{c}^{2 n-1}, \quad \Delta \tau:=2 \Delta t \quad \text { and } \quad \Lambda^{n}:=\frac{J^{2 n+1}-J^{2 n-3}}{4 \Delta t} .
$$

then collecting $(7.4 \mathrm{a}),(7.4 \mathrm{~d})$ and $(7.6)$, one can show that the following system holds

$$
\left\{\begin{array}{l}
M_{\sigma, c} \frac{U_{c}^{n+1}-2 U_{c}^{n}+U_{c}^{n-1}}{\Delta \tau^{2}}+K_{c} U_{c}^{n}-C_{c}^{*} \Lambda^{n}=0, \\
M_{\sigma, f} \frac{U_{f}^{n+1}-2 U_{f}^{n}+U_{f}^{n-1}}{\Delta \tau^{2}}+\left[I_{f}-\frac{\Delta \tau^{2}}{16} K_{f} M_{\sigma, f}^{-1}\right]\left[K_{f} U_{f}^{n}-C_{f}^{*} \Lambda^{n}\right]=0 \\
C_{c} U_{c}^{n+1}+C_{f} U_{f}^{n+1}=0 .
\end{array}\right.
$$


This new formulation of system (7.4) shows that the local time stepping proposed in [17] is in fact equivalent to the scheme developed in Section 4.2 (compare the above equations with (4.6)). Therefore the local time stepping proposed in [17] can be seen as a transmission problem between two second order schemes, one of which having a relaxed stability condition by adding stabilising terms. The computational burden of the schemes we propose in Section 4.3 is equivalent to the one of the schemes proposed in [17]. In fact we conjecture that, the local time stepping of [17] can be recast in the formalism of Section 4.3 with $\mathcal{P}_{p} \equiv \widetilde{\mathcal{P}}_{p, \ell}$. It has to be noted that the schemes in [17] are not proven to be second order convergent (in space and time) for the $H^{1}$-norm which is in accordance with the convergence results of Section 5.3.

\section{Conclusions}

In this work we have presented and analysed a family of second order in time discretisation strategy for linear wave equations. We have shown that they correspond to either locally implicit schemes or to local time stepping. For the analysis we have considered the case of smooth solutions. Then, we have shown that, if a well-defined stability condition holds, then second order space-time convergence property holds in the context of abstract Galerkin approximations of the wave equation. Finally, we have presented 1D numerical convergence results that confirm the obtained theoretical results. As far as local time stepping strategies are concerned, after comparisons with existing methods we have confirmed the interest of the proposed approach since, in terms of accuracy, it yields second order convergence results in the $H^{1}$-norm (contrary to $\left.[17,19]\right)$ and in terms of computational cost, it is equivalent to the method proposed in [17].

\section{Appendix A. Convergence Result for the SEMI-Discrete Problem}

In Section 2.1 we have assumed that the solution to the continuous problem satisfies some extra-regularity in time. One can expect that, in general, existence and uniqueness results of solutions for source term and initial data do not immediately provide the adequate time regularity. Such extra regularity can be obtained in several ways. A simple one is to assume that initial data are null and the source term is smooth and vanishes at the initial time. More precisely, let $m \in \mathbb{N}^{*}$ and assume $\boldsymbol{f} \in W^{m, 1}(0, T ; \boldsymbol{H})$ and $\boldsymbol{f}^{(n)}(0)=\mathbf{0}$ for $m \in \mathbb{N}$ such that $n<m$, then it is expected that the unique solution of Problem 2.4 with null initial data belongs to

$$
(\boldsymbol{u}, \lambda) \in C^{m+1}([0, T] ; \boldsymbol{H}) \cap C^{m}([0, T] ; \boldsymbol{V}) \times C^{m-1}([0, T] ; L) .
$$

Such result is obtained by straightforward differentiation in time of the variational formulation. If initial data do not vanish then the question of the regularity of solution in time is more intricate since the initial data should be sufficiently smooth in space (or in a abstract framework, it should belong to the domain of some unbounded operator). Moreover, the same question arises at the semi discrete level, since it is not clear that the continuous result in general transfers to the semi-discrete setting.

Existence and uniqueness results for the semi-discrete problem are generally a direct consequence of the results obtained at the continuous level, and the uniform estimates of Assumption 2.2 are obtained by energy techniques.

To obtain smooth semi-discrete solutions one can assume again that initial data are null and the source term is smooth and vanishes at the initial time, then one can define the source term for the semi-discrete problem as follows: the source term $\boldsymbol{f}_{h}(t)$ is chosen at every time $t$ as the orthogonal projection of $\boldsymbol{f}(t)$ in $\boldsymbol{V}_{h}$ with respect to the scalar product of $\boldsymbol{H}$, i.e., $\boldsymbol{f}_{h}(t) \in \boldsymbol{V}_{h}$ and

$$
\left(\boldsymbol{f}_{h}(t), \boldsymbol{v}_{h}\right)=\left(\boldsymbol{f}(t), \boldsymbol{v}_{h}\right), \quad \boldsymbol{v}_{h} \in \boldsymbol{V}_{h} .
$$

From this definition we deduce that if $\boldsymbol{f} \in W^{m, 1}(0, T ; \boldsymbol{H})$ then $\boldsymbol{f}_{h} \in W^{m, 1}\left(0, T ; \boldsymbol{V}_{h}\right)$ and

$$
\sum_{n=0}^{m} \int_{0}^{T}\left|\frac{\mathrm{d}^{n}}{\mathrm{~d} t^{n}} \boldsymbol{f}_{h}(t)\right| \mathrm{d} t \leq \sum_{n=0}^{m} \int_{0}^{T}\left|\frac{\mathrm{d}^{n}}{\mathrm{~d} t^{n}} \boldsymbol{f}(t)\right| \mathrm{d} t .
$$


Then, one can prove that the semi-discrete solution is smooth in time by first differentiating in time the semi-discrete variational formulation and second, by using the standard existence, uniqueness and regularity results for wave propagation problems for the successive time derivatives of the solution.

For the sake of completeness we now give the proof of Theorem 2.3 that is rather standard and is inspired from the one given in [1].

Proof of Theorem 2.3. By assumption there exists a solution to (2.3) such that (2.7) holds (or (A.1) with $m=3$ ). Then, System (2.8) can be recast as: find $\left(\boldsymbol{u}_{h}(t), \lambda_{h}(t)\right) \in \boldsymbol{V}_{h} \times L_{h}$ solution to

$$
\begin{cases}\frac{\mathrm{d}^{2}}{\mathrm{~d} t^{2}}\left(\boldsymbol{u}_{h}, \boldsymbol{v}_{h}\right)+a\left(\boldsymbol{u}_{h}, \boldsymbol{v}_{h}\right)+b\left(\boldsymbol{v}_{h}, \lambda_{h}\right)=\left(\boldsymbol{f}_{h}, \boldsymbol{v}_{h}\right) & \boldsymbol{v}_{h} \in \boldsymbol{V}_{h}, \\ b\left(\boldsymbol{u}_{h}, \mu_{h}\right)=0 & \mu_{h} \in \boldsymbol{L}_{h},\end{cases}
$$

We introduce the elliptic projection $\hat{\boldsymbol{u}}_{h}(t)$ of $\boldsymbol{u}(t)$ defined as: for $(\boldsymbol{u}(t), \lambda(t)) \in \boldsymbol{V} \times L$ find $\left(\hat{\boldsymbol{u}}_{h}(t), \hat{\lambda}(t)\right) \in \boldsymbol{V}_{h} \times L_{h}$ such that, for some $C_{a}>0$ sufficiently large,

$$
\begin{cases}C_{a}\left(\hat{\boldsymbol{u}}_{h}-\boldsymbol{u}, \boldsymbol{v}_{h}\right)+a\left(\hat{\boldsymbol{u}}_{h}-\boldsymbol{u}, \boldsymbol{v}_{h}\right)+b\left(\boldsymbol{v}_{h}, \hat{\lambda}_{h}-\lambda\right)=0 & \boldsymbol{v}_{h} \in \boldsymbol{V}_{h}, \\ b\left(\hat{\boldsymbol{u}}_{h}-\boldsymbol{u}, \mu_{h}\right)=0 & \mu_{h} \in \boldsymbol{L}_{h} .\end{cases}
$$

Notice that $\hat{\boldsymbol{u}}_{h}$ can be seen as the solution to a static problem of mixed type and depends on the time $t$ only because the terms $\boldsymbol{u}$ and $\lambda$ do so. Therefore, $\hat{\boldsymbol{u}}_{h}$ inherits directly from $\boldsymbol{u}$ and $\lambda$ its regularity in the parameter $t$.

Since the inf-sup condition (2.10) is satisfied and because of the ellipticity and continuity of $a(\cdot, \cdot)$ (see Eq. (2.2)) one can use standard results on mixed problem - see [6] - to show that for all $t \in[0, T]$, there exists $C$ independent of $h$ such that, for $m \in\{0,1,2\}$,

$$
\left\|\frac{\mathrm{d}^{m}}{\mathrm{~d} t^{m}} \hat{\boldsymbol{u}}_{h}-\frac{\mathrm{d}^{m}}{\mathrm{~d} t^{m}} \boldsymbol{u}\right\| \leq C\left(\inf _{\boldsymbol{v}_{h} \in \boldsymbol{V}_{h}}\left\|\boldsymbol{v}_{h}-\frac{\mathrm{d}^{m}}{\mathrm{~d} t^{m}} \boldsymbol{u}\right\|+\inf _{\mu_{h} \in L_{h}}\left\|\mu_{h}-\frac{\mathrm{d}^{m}}{\mathrm{~d} t^{m}} \lambda\right\|_{L}\right) .
$$

The strategy is now to obtain an estimation of $\left\|\boldsymbol{u}_{h}-\hat{\boldsymbol{u}}_{h}\right\|$. One can show, using (A.3), (2.4) and (A.4) that

$$
\left\{\begin{aligned}
\frac{\mathrm{d}^{2}}{\mathrm{~d} t^{2}}\left(\boldsymbol{u}_{h}-\hat{\boldsymbol{u}}_{h}, \boldsymbol{v}_{h}\right)+a\left(\boldsymbol{u}_{h}-\hat{\boldsymbol{u}}_{h}, \boldsymbol{v}_{h}\right)+b\left(\boldsymbol{v}_{h}, \lambda_{h}-\hat{\lambda}_{h}\right) & \\
=-\frac{\mathrm{d}^{2}}{\mathrm{~d} t^{2}}\left(\hat{\boldsymbol{u}}_{h}-\boldsymbol{u}, \boldsymbol{v}_{h}\right)+\left(\boldsymbol{f}_{h}-\boldsymbol{f}, \boldsymbol{v}_{h}\right)+C_{a}\left(\hat{\boldsymbol{u}}_{h}-\boldsymbol{u}, \boldsymbol{v}_{h}\right) & \boldsymbol{v}_{h} \in \boldsymbol{V}_{h} \\
b\left(\boldsymbol{u}_{h}-\hat{\boldsymbol{u}}_{h}, \mu_{h}\right)=0 & \mu_{h} \in \boldsymbol{L}_{h}
\end{aligned}\right.
$$

By standard energy estimates, one can show that there exists $C$ independent of $T$ and $h$ such that

$$
\begin{aligned}
\sup _{t \in[0, T]} & \left(\left|\frac{\mathrm{d}}{\mathrm{d} t} \boldsymbol{u}_{h}(t)-\frac{\mathrm{d}}{\mathrm{d} t} \hat{\boldsymbol{u}}_{h}(t)\right|^{2}+a\left(\boldsymbol{u}_{h}(t)-\hat{\boldsymbol{u}}_{h}(t), \boldsymbol{u}_{h}(t)-\hat{\boldsymbol{u}}_{h}(t)\right)\right)^{\frac{1}{2}} \\
\leq & \left(\left|\boldsymbol{u}_{1, h}-\frac{\mathrm{d}}{\mathrm{d} t} \hat{\boldsymbol{u}}_{h}(0)\right|^{2}+a\left(\boldsymbol{u}_{0, h}-\hat{\boldsymbol{u}}_{h}(0), \boldsymbol{u}_{0, h}-\hat{\boldsymbol{u}}_{h}(0)\right)\right)^{\frac{1}{2}} \\
& +C \int_{0}^{T}\left(\left|\hat{\boldsymbol{u}}_{h}(t)-\boldsymbol{u}(t)\right|+\left|\frac{\mathrm{d}^{2}}{\mathrm{~d} t^{2}} \hat{\boldsymbol{u}}_{h}(t)-\frac{\mathrm{d}^{2}}{\mathrm{~d} t^{2}} \boldsymbol{u}(t)\right|+\left|\boldsymbol{f}_{h}(t)-\boldsymbol{f}(t)\right|\right) \mathrm{d} t .
\end{aligned}
$$


This estimate can be further simplified by deducing first a control in the norm $|\cdot|$ of the discrepancy which is obtained thanks to the following estimation, for all $t \in[0, T]$,

$$
\left|\boldsymbol{u}_{h}(t)-\hat{\boldsymbol{u}}_{h}(t)\right| \leq\left|\boldsymbol{u}_{h, 0}-\hat{\boldsymbol{u}}_{h}(0)\right|+T \sup _{t \in[0, T]}\left|\frac{\mathrm{d}}{\mathrm{d} t} \boldsymbol{u}_{h}(t)-\frac{\mathrm{d}}{\mathrm{d} t} \hat{\boldsymbol{u}}_{h}(t)\right|,
$$

second, by introducing another scalar $C$ independent of $T$ and $h$ such that,

$$
a\left(\boldsymbol{u}_{0, h}-\hat{\boldsymbol{u}}_{h}(0), \boldsymbol{u}_{0, h}-\hat{\boldsymbol{u}}_{h}(0)\right) \leq C\left\|\boldsymbol{u}_{0, h}-\hat{\boldsymbol{u}}_{h}(0)\right\|^{2}
$$

and third using the ellipticity of the bilinear form $a(\cdot, \cdot)$. We obtain,

$$
\begin{aligned}
\sup _{t \in[0, T]}\left\|\boldsymbol{u}_{h}(t)-\hat{\boldsymbol{u}}_{h}(t)\right\| \leq & C\left(\left|\boldsymbol{u}_{1, h}-\frac{\mathrm{d}}{\mathrm{d} t} \hat{\boldsymbol{u}}_{h}(0)\right|+\left\|\boldsymbol{u}_{0, h}-\hat{\boldsymbol{u}}_{h}(0)\right\|\right) \\
& +C \sup _{t \in[0, T]}\left(\left|\hat{\boldsymbol{u}}_{h}(t)-\boldsymbol{u}(t)\right|+\left|\frac{\mathrm{d}^{2}}{\mathrm{~d} t^{2}} \hat{\boldsymbol{u}}_{h}(t)-\frac{\mathrm{d}^{2}}{\mathrm{~d} t^{2}} \boldsymbol{u}(t)\right|+\left|\boldsymbol{f}_{h}(t)-\boldsymbol{f}(t)\right|\right),
\end{aligned}
$$

for another positive scalar $C$ independent of and $h$. Finally, the result of the theorem is a consequence of (A.5) and the triangle inequality that is used after writing

$$
\boldsymbol{u}_{h}-\hat{\boldsymbol{u}}_{h}=\left(\boldsymbol{u}_{h}-\boldsymbol{u}\right)+\left(\boldsymbol{u}-\hat{\boldsymbol{u}}_{h}\right) .
$$

Note that a similar decomposition is used to deal with the estimation of the initial conditions.

\section{Appendix B. Proof of the discrete Gronwall's lemma}

The purpose of this section is to provide a proof of the discrete Gronwall's lemma (3.42). Let

$$
w^{m-1}:=A+B \sum_{n=1}^{m-1}\left(v^{n}\right)^{1 / 2} .
$$

Suppose that for all $m \geq 1$,

$$
v^{m-1} \leq w^{m-1}
$$

(i) Suppose that $w^{m-1}=0$ then $v^{m-1}=0$ which proves the result.

(ii) Suppose that $w^{m-1} \neq 0$. Then

$$
w^{m}-w^{m-1}=B\left(v^{m}\right)^{1 / 2} \leq B\left(w^{m}\right)^{1 / 2}
$$

by hypothesis. Then

$$
\left(\left(w^{m}\right)^{1 / 2}-\left(w^{m-1}\right)^{1 / 2}\right)\left(\left(w^{m}\right)^{1 / 2}+\left(w^{m-1}\right)^{1 / 2}\right) \leq B\left(\left(w^{m}\right)^{1 / 2}+\left(w^{m-1}\right)^{1 / 2}\right)
$$

since $w^{m-1} \geq 0$. Therefore

$$
\left(w^{m}\right)^{1 / 2} \leq B+\left(w^{m-1}\right)^{1 / 2}
$$

By summation we get

$$
\left(v^{m}\right)^{1 / 2} \leq\left(w^{m}\right)^{1 / 2} \leq\left(w^{0}\right)^{1 / 2}+m B .
$$

Since $w^{0}=A$ we get the expected result.

Acknowledgements. The authors would like to thank Prof. Dr. Marlis Hochbruck and Dr. Andreas Sturm for their helpful discussions at the Conference on Mathematics of Wave Phenomena at KIT in July 2018. 


\section{REFERENCES}

[1] J. Albella, H. Ben Dhia, S. Imperiale and J. Rodríguez, Mathematical and numerical study of transient wave scattering by obstacles with a new class of Arlequin coupling. SIAM J. Numer. Anal. 57 (2019) 2436-2468.

[2] E. Bécache and P. Joly, Space-time mesh refinement for elastodynamics, Comput. Methods Appl. Mech. Eng. 194 (2005) $355-366$.

[3] E. Bécache, J. Rodríguez and C. Tsogka, Convergence results of the fictitious domain method for a mixed formulation of the wave equation with a Neumann boundary condition. ESAIM: M2AN 43 (2009) 377-398.

[4] F. Ben Belgacem, C. Bernardi, N. Chorfi and Y. Maday, Inf-sup conditions for the mortar spectral element discretization of the Stokes problem. Numer. Math. 85 (2000) 257-281.

[5] D. Boffi, F. Brezzi and M. Fortin, Mixed Finite Element Methods and Applications. Springer Science (2013).

[6] F. Brezzi and M. Fortin, Mixed and Hybrid Finite Element Methods. Springer Science (2012) 15.

[7] J. Chabassier and S. Imperiale, Fourth order energy-preserving locally implicit time discretisation for linear wave equations. Int. J. Numer. Methods Eng. 106 (2016) 593-622.

[8] J. Chabassier and S. Imperiale, Space/Time convergence analysis of a class of conservative schemes for linear wave equations. C.R. Math. 355 (2017) 282-289.

[9] J. Chabassier, J. Diaz and S. Imperiale, Construction and analysis of fourth order, energy consistent, family of explicit time discretizations for dissipative linear wave equations. ESAIM: M2AN 54 (2020) 845-878.

[10] G. Cohen, Higher-order Numerical Methods for Transient Wave Equations. Springer-Verlag (2001).

[11] G. Cohen, P. Joly and N. Tordjman, Higher-order finite elements with mass-lumping for the 1D wave equation. Finite Elem. Anal. Des. 16 (1994) 329-336.

[12] G. Cohen, P. Joly, J.E. Roberts and N. Tordjman, Higher-order triangular finite elements with mass lumping for the wave equation. SIAM: J. Numer. Anal. 38 (2001) 2047-2078.

[13] F. Collino, T. Fouquet and P. Joly, A conservative space-time mesh refinement method for the 1-d wave equation. Part I: Construction, Numer. Math. 95 (2003) 197-221.

[14] F. Collino, T. Fouquet and P. Joly, A conservative space-time mesh refinement method for the 1-D wave equation. II. Analysis. Numer. Math. 95 (2003) 223-251.

[15] C. Constantin, M. Hochbruck and A. Sturm, On leapfrog-Chebyshev schemes. SIAM J. Numer. Anal. 58 (2020) $2404-2433$.

[16] R. Dautray and J.-L. Lions, Mathematical Analysis and Numerical Methods for Science and Technology - Volume 5 and 6 Evolution Problems I and II. Springer-Verlag Berlin (2000).

[17] G. Derveaux and P. Joly and J. Rodríguez, Effective Computational Methods for Wave Propagation. Chap 13: Space Time Mesh Refinement Methods. Chapman and Hall/CRC (2008).

[18] S. Descombes, S. Lanteri and L. Moya, Locally implicit time integration strategies in a discontinuous Galerkin method for Maxwell's equations. J. Sci. Comput. 56 (2013) 190-218.

[19] J. Diaz and M.J. Grote, Energy conserving explicit local time-stepping for second-order wave equations. SIAM J. Sci. Comput. 31 (2009) 1985-2014.

[20] J. Diaz and M.J. Grote, Multi-level explicit local time-stepping methods for second-order wave equations. Comput. Methods Appl. Mech. Eng. 291 (2015) 240-265.

[21] V. Dolean, H. Fahs, L. Fezoui and S. Lanteri, Locally implicit discontinuous Galerkin method for time domain electromagnetics. J. Comput. Phys. 229 (2010) 512-526.

[22] M. Dumbser, M. Käser and E.F. Toro, An arbitrary high-order Discontinuous Galerkin method for elastic waves on unstructured meshes - V. Local time stepping and p-adaptivity. Geophys. J. Int. 171 (2007) 695-717.

[23] M. Durufle, P. Grob and P. Joly, Influence of Gauss and Gauss-Lobatto quadrature rules on the accuracy of a quadrilateral finite element method in the time domain. Numer. Methods Part. Differ. Equ. 25 (2009) 526-551.

[24] J.C. Gilbert and P. Joly, Higher order time stepping for second order hyperbolic problems and optimal CFL conditions. Part. Differ. Equ. 16 (2008) 67-93.

[25] M.J. Grote and T. Mitkova, Explicit local time-stepping methods for Maxwell's equations. J. Comput. Appl. Math. 234 (2010) 3283-3302.

[26] M.J. Grote, M. Mehlin and S. Sauter, Convergence analysis of energy conserving explicit local time-stepping methods for the wave equation. SIAM J. Numer. Anal. 56 (2018) 994-1021.

[27] M.J. Grote, S. Michel and S. Sauter, Stabilized leapfrog based local time-stepping method for the wave equation. Preprint: arXiv:2005.13350 (2021).

[28] P. Hauret and P. Le Tallec, A discontinuous stabilized mortar method for general 3D elastic problems. Comput. Methods Appl. Mech. Eng. 196 (2007) 4881-4900.

[29] J.S. Hesthaven and T. Warburton, Nodal Discontinuous Galerkin Methods: Algorithms, Analysis, and Applications. Springer Science \& Business Media (2007).

[30] M. Hochbruck and A. Sturm, Error analysis of a second-order locally implicit method for linear Maxwell's equations. SIAM J. Numer. Anal. 54 (2016) 3167-3191.

[31] M. Hochbruck and A. Sturm, Upwind discontinuous Galerkin space discretisation and locally implicit time integration for linear Maxwell's equations. Math. Comput. 88 (2019) 1121-1153.

[32] W. Hundsdorfer and J.G. Verwer, Numerical solution to Time-Dependent Advection-Diffusion-Reaction Equations, Springer Series in Computational Mathematics (2003). 
[33] P. Joly and J. Rodríguez, An error analysis of conservative space-time mesh refinement methods for the one-dimensional wave equation. SIAM J. Numer. Anal. 43 (2005) 825-859.

[34] P. Joly and J. Rodríguez, Optimized higher order time discretisation of second order hyperbolic problems: construction and numerical study. J. Comput. Appl. Math. 234 (2010) 1953-1961.

[35] Y. Maday and A.T. Patera, Spectral element methods for the incompressible Navier-Stokes equations. In: State-of-the-art Surveys On Computational Mechanics. American Society of Mechanical Engineers (1989).

[36] Y. Maday, C. Mavriplis and A.T. Patera, Nonconforming mortar element methods: application to spectral discretisations. In: Domain Decomposition Methods. SIAM Philadelphia (1989) 392-418.

[37] J. Rodríguez, Une nouvelle méthode de raffinement de maillage spatio-temporel pour l'équation des ondes. C. R. Math. Acad. Sci. Paris 339 (2004) 445-450.

[38] J. Rodríguez, A spurious-free space-time mesh refinement for elastodynamics. Int. J. Multiscale Comput. Eng. 6 (2008) 263279.

[39] T. Rylander, Stability of Explicit-Implicit Hybrid Time-Stepping Schemes for Maxwell's Equations. J. Comput. Phys. 179 (2002) 426-438.

[40] P.J. van Der Houwen and B.P. Sommeijer, On the internal stability of explicit, $m$-Stage Runge-Kutta methods for large m-Values. J. Appl. Math. Mech. 60 (1980) 479-485.

[41] B.I. Wohlmuth, A mortar finite element method using dual spaces for the lagrange multiplier. SIAM J. Numer. Anal. 38 (2000) 989-1012.

[42] B.I. Wohlmuth, Discretization Methods and Iterative Solvers Based on Domain Decomposition. In Vol. 17 of Lecture Notes in Computational Science and Engineering. Springer (2001).

[43] https://eigen.tuxfamily.org/.

[44] https://gitlab.inria.fr/local-schemes/supplementary-sources.

\section{Subscribe to Open (S2O)}

\section{A fair and sustainable open access model}

This journal is currently published in open access with no charge for authors under a Subscribe-to-Open model (S2O). Open access is the free, immediate, online availability of research articles combined with the rights to use these articles fully in the digital environment.

$\mathrm{S} 2 \mathrm{O}$ is one of the transformative models that aim to move subscription journals to open access. Every year, as long as the minimum amount of subscriptions necessary to sustain the publication of the journal is attained, the content for the year is published in open access.

\section{Ask your library to support open access by subscribing to this $\mathrm{S} 2 \mathrm{O}$ journal.}

Please help to maintain this journal in open access! Encourage your library to subscribe or verify its subscription by contacting subscribers@edpsciences.org

We are thankful to our subscribers and sponsors for making it possible to publish the journal in open access, free of charge for authors. More information and list of sponsors: https://www.edpsciences.org/en/maths-s2o-programme 